\title{
Dispersion controlled highly nonlinear fibers for all optical processing at telecoms wavelengths
}

\author{
Xian Feng *, Francesco Poletti, Angela Camerlingo, Francesca Parmigiani, Periklis Petropoulos, \\ Peter Horak, Giorgio M. Ponzo, Marco Petrovich, Wei H. Loh and David J. Richardson \\ Optoelectronics Research Centre, University of Southampton, Southampton, SO17 1BJ, UK \\ *Corresponding author: xif@orc.soton.ac.uk
}

Keywords: non-silica glass fiber; microstructured optical fiber; highly nonlinear fiber; chromatic dispersion control; all optical processing

\begin{abstract}
We review our recent progress in the development of lead silicate glass fibers with high nonlinearity and tailored near-zero dispersion at telecommunication wavelengths, encompassing holey, all-solid microstructured and W-type fiber designs. The fabrication techniques and relative merits of each fiber design are described in detail. The optical properties of the fabricated fibers are assessed both experimentally and through accurate numerical simulations. The significant potential of lead silicate highly nonlinear fibers for all-optical signal processing at telecommunication wavelengths is shown via a number of key experimental demonstrators.
\end{abstract}

\section{Introduction}

Highly Nonlinear Fibers (HNLFs) with tailored chromatic dispersion are of extreme interest for many photonic applications, ranging from supercontinuum generation to all-optical signal manipulation and optical parametric devices. Generally, supercontinuum generation applications require a shifted chromatic dispersion profile, so that the zero-dispersion wavelength of the nonlinear fiber is accurately positioned to match that of the pump source. This allows the relative strength of nonlinear effects to be maximized and can give rise to extreme spectral broadening even in a short fiber length [1]. Optical signal processing applications on the other hand, such as wavelength conversion which is a crucial operation in high speed wavelength division multiplexed (WDM) optical networks, often require fibers with even tighter specifications. Fourwave mixing (FWM) in fibers in particular, is regarded as one of the most promising wavelength conversion mechanisms, due to its transparency in terms of both modulation format and bit rate. By combining a strong pump wave at an angular frequency $\left(\omega_{\mathrm{p}}\right)$ with a signal at another frequency $\left(\omega_{\mathrm{s}}\right)$ in a highly nonlinear fiber, parametric gain can be achieved [2]. At the same time, a converted idler $\left(\omega_{\mathrm{i}}\right)$ will be generated at the frequency $\omega_{\mathrm{i}}=2 \omega_{\mathrm{p}}-\omega_{\mathrm{s}}$. In order to obtain efficient and broadband FWM, it is necessary to phase-match the waves involved in the process along the full length of the fiber. Therefore, fibers that combine a high effective nonlinear coefficient with low dispersion, low dispersion slope and a short propagation length are typically required for such applications.

The effective nonlinear coefficient $\gamma$ of a fiber is the primary parameter used in order to gauge its performance for nonlinear device applications, and can be expressed as:

$\gamma=2 \pi n_{2} /\left(\lambda A_{e f f}\right)$ 
where $\lambda$ is the wavelength of light, $A_{\text {eff }}$ is the effective mode area, and $n_{2}$ is the nonlinear refractive index of the glass, respectively. According to Eq.(1), a high nonlinearity $\gamma$ can be achieved by choosing a high $\mathrm{n}_{2}$ glass as the host material and/or by targeting the smallest possible effective mode area $\mathrm{A}_{\mathrm{eff}}$ (which is typically obtained in a wavelength-sized fiber core with sufficiently large index contrast with the cladding). The second key optical property of a fiber is its dispersion profile, which characterizes the wavelength dependence of the group velocity of the guided mode. The total dispersion (D) of a fiber can be expressed, to very good approximation, as the sum of the material dispersion $\left(D_{m}\right)$ (see Fig.1(a)) and the waveguide dispersion $\left(\mathrm{D}_{\mathrm{w}}\right)$, i.e., $D=D_{m}+D_{w}$. For many parametric-based telecommunications devices one would ideally like to design the waveguide dispersion in such a way that it exactly compensates the material dispersion over a broad spectral range, to generate a perfect dispersionless fiber, as schematically shown in Fig.1(b). Although a wideband dispersion-less behavior is extremely challenging to achieve in practice, broadband, near-zero dispersion profiles have been obtained by exploiting the novel holey or microstructured fiber designs discussed here.

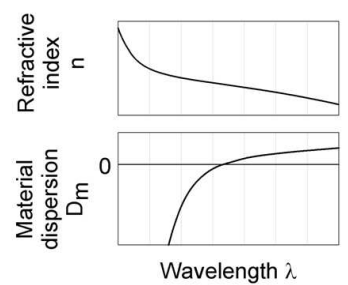

(a)

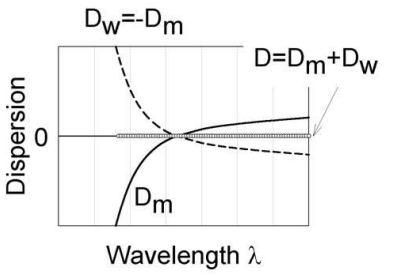

(b)

Fig. 1. (a) Schematic example of the material dispersion of an optical glass; the wavelength scale shown would normally span over several 100 s of nanometers. (b) Ideal waveguide dispersion $\left(D_{w}=-D_{m}\right)$ for achieving a flat and near-zero dispersion profile over a broad wavelength range.

In this work, we review our progress towards the design and fabrication of a highly nonlinear dispersion-flattened optical fiber based on high-index lead silicate glass and designed for operation at around $1.55 \mu \mathrm{m}$. Material aspects are discussed as well as the relative merits of various optimized fibers. The first promising results on the use of our fibers in short-length fiber based nonlinear devices for all optical processing will also be discussed.

\section{Structure and material aspects for dispersion-tailored highly nonlinear fibers}

\subsection{Fiber structures}

Over the past decade and a half [3, 4], single-material holey fibers (HFs), often also referred to as photonic crystal fibers (PCFs) or microstructured optical fibers (MOFs), have attained considerable technological maturity. These fibers open up the possibility for much higher nonlinearity and better flexibility for dispersion control than is possible using conventional fiber technology, particularly for realizing flat, near-zero dispersion profiles over a broad wavelength range. HFs exploit the large index contrast between air and glass, while taking full advantage of the design flexibility of wavelength-scale features in their 'holey' or microstructured cladding.

Fig. 2 shows the schematics of four types of typical index-guided nonlinear optical fibers: (a) HF with an air-suspended core (ASC), (b) HF with a complex two dimensional (2D) microstructured cladding, and (c) MOF with an all-solid one-dimensional (1D) microstructured cladding. The fourth design (Fig.2d) is not typically classified as a MOF and comprises a W-type step-index profile with high index-contrast; this design is quite common in the development of germanium-doped silica highly nonlinear fibers. In the following sections the fiber design, fabrication techniques and fiber characterization will be discussed in detail for each fiber type. Some key applications achieved with these fibers will also be discussed. 


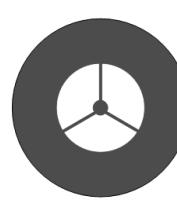

(a)

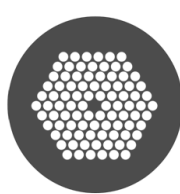

(b)

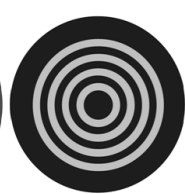

(c)

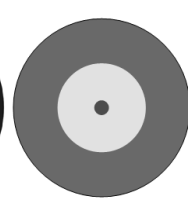

(d)

Fig. 2. (a) ASC HF; (b) HF with complex two dimensional (2D) microstructured cladding; (c) all-solid 1D MOF; and (d) W-type step-index profile fiber with high index-contrast.

\subsection{Fiber materials}

Eq.(1) indicates that using a glass with high $\mathrm{n}_{2}[5,6]$ will result in a higher fiber effective nonlinearity, $\gamma$. According to material chemistry, the linear refractive index, $n$, and the nonlinear refractive index, $\mathrm{n}_{2}$, of a dielectric material are both attributed to the polarizability and the hyperpolarizability of the constituent chemical ions [7-9]. Thus glasses consisting of ions with heavy atomic weight and/or large ionic radii will generally exhibit high values of $n$ and $n_{2}$. Fig. 3 illustrates the relation between $\mathrm{n}$ and $\mathrm{n}_{2}$ of various optical glasses, including fluoride glasses, silica, lead silicate glasses, tellurite glasses, other heavy metal oxide (HMO) glasses, and chalcogenide glasses. It can be seen that the nonlinear refractive index $\mathrm{n}_{2}$ increases with the linear refractive index $\mathrm{n}$, in agreement with the empirical prediction of Miller's rule: the nonlinear optical response of a material is related to its linear response. In particular, high-index HMO glasses and chalcogenide glasses (based on $\mathrm{S}$, Se, and Te) possess a nonlinear refractive index, $\mathrm{n}_{2}$, which is $1-3$ orders of magnitude higher than silica glass $\left(2.5 \times 10^{-20} \mathrm{~m}^{2} / \mathrm{W}\right)$. This indicates that by choosing a high-index glass as the host material for a nonlinear fiber, the fiber nonlinearity $\gamma$ can be enhanced by 1-3 orders of magnitude. In addition, glasses of higher refractive indices have higher glass/air index contrast, thus allowing for better mode confinement in the core and smaller effective areas to be achieved. Consequently, the maximum nonlinearity $\gamma$ of a holey fiber based on a high-index glass can be as high as $10^{3}-10^{5}$ times higher than conventional silica fibers. The use of such fibers opens up the possibility for extremely efficient and compact nonlinear fiber devices with meter and sub-meter lengths.

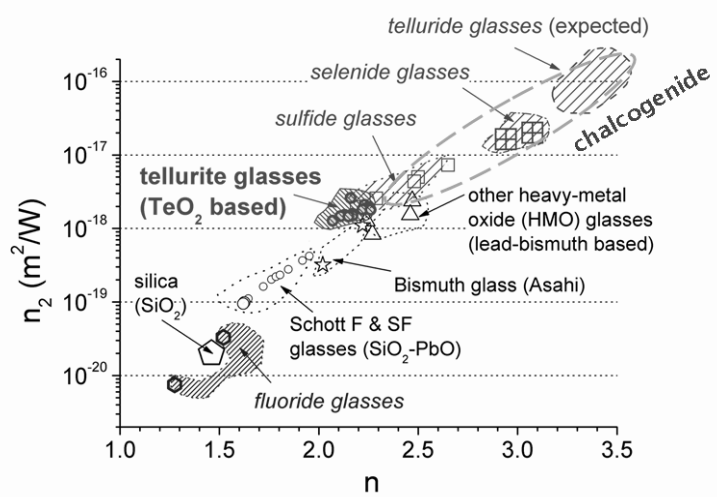

Fig. 3. Relation between the linear $(n)$ and nonlinear refractive index $\left(\mathrm{n}_{2}\right)$ of various optical glasses

\subsection{Understanding refractive index and material dispersion of optical glass}

When an electromagnetic wave at optical frequencies interacts with the bound electrons of a dielectric medium, such as a glass, the linear response of the medium in general depends on the optical frequency, $\omega$. This effect, often referred to as chromatic dispersion of the dielectric material, is characterized by a frequency- (or wavelength-) dependent refractive index, $\mathrm{n}(\omega)$ (or $\mathrm{n}(\lambda)$ ). On a fundamental level, the origin of chromatic dispersion is related to the characteristic 
resonance frequencies at which the medium absorbs the electromagnetic radiation through oscillations of bound electrons. At wavelengths far from these resonances, the refractive index can be approximately expressed by the well-known Sellmeier equation [10]:

$$
n^{2}(\varpi)=1+\sum_{i=1}^{m} \frac{B_{i} \varpi_{i}^{2}}{\varpi_{i}^{2}-\varpi^{2}},
$$

where $\omega_{i}$ is the resonance frequency and $B_{i}$ is the strength of $i$-th resonance. In practice, it is very difficult to precisely determine all the resonant frequencies, and an approximate model is often used, which is based on an average electronic absorption bandgap and an average lattice absorption bandgap. This leads to the simplified two-pole Sellmeier equation [10]:

$$
n^{2}(\varpi)=1+\frac{B_{1} \varpi_{1}^{2}}{\varpi_{1}^{2}-\varpi^{2}}+\frac{B_{2} \varpi_{2}^{2}}{\varpi_{2}^{2}-\varpi^{2}},
$$

where $\omega_{1}$ is the resonance absorption frequency of the electronic transition, $\omega_{2}$ is the resonance absorption frequency of the vibration transition, and $\omega_{1}$ and $\omega_{2}$ typically lie in the ultraviolet (UV) and in the infrared (IR) region, respectively. $B_{1}$ and $B_{2}$ are the corresponding resonance strengths. Eq. (3) can be formulated as a function of the wavelength $\lambda$ :

$$
n^{2}(\lambda)=A+\frac{B \lambda^{2}}{\lambda^{2}-\lambda_{1}^{2}}+\frac{C \lambda^{2}}{\lambda^{2}-\lambda_{2}^{2}} \text {, }
$$

where $\lambda_{1}$ and $\lambda_{2}$ are the absorption wavelengths in the UV and IR regions, respectively; A, B, C are the constants concerned with the absorption strength, and $\lambda_{1,2}=2 \pi \mathrm{c} / \omega_{1,2}[11]$.

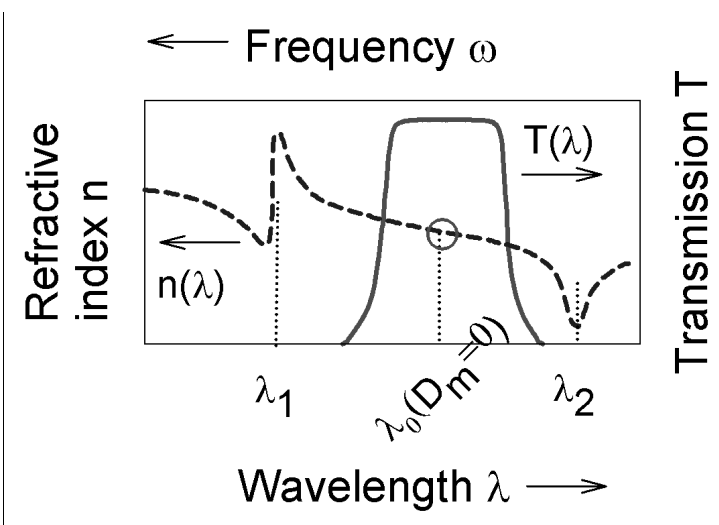

Fig. 4 Schematic dispersion curve of refractive index $n(\lambda)$ and transmission curve $T(\lambda)$ of a dielectric material in the optical wavelength range.

Fig. 4 shows a schematic of the refractive index $n(\lambda)$ and of the transmission $T(\lambda)$ of a dielectric material at optical frequencies. The material dispersion is then defined as:

$$
D_{m}=-\frac{\lambda}{c} \cdot \frac{d^{2} n(\lambda)}{d \lambda^{2}}
$$

According to Eq.(5), the wavelength of zero material dispersion, $\lambda_{0}$, falls at the inflection point of $n(\lambda)$, indicated by the circle in Fig.4. From the viewpoint of material science, the position of $\lambda_{0}$ is determined by the combined effect of both the average electronic absorption bandgap and the average lattice absorption bandgap, as modeled by the two-pole Sellmeier equation, Eq.(4). Table 1 shows a summary of $\lambda_{0}, \lambda_{1}$ and $\lambda_{2}$ for various optical glasses. It is clear that the position of $\lambda_{0}$ depends on the positions of the resonance peaks in the UV and the IR, $\lambda_{1}$ and $\lambda_{2}$, as well as on the refractive index. For most high-index, high-nonlinearity glasses such as lead/bismuth 
silicate glasses (HMO, $\mathrm{SiO}_{2}$ based) and pure $\mathrm{HMO}$ glasses (non- $\mathrm{SiO}_{2}$ based), including tellurite $\left(\mathrm{TeO}_{2}\right.$ based), germanate $\left(\mathrm{GeO}_{2}\right.$ based) and gallate $\left(\mathrm{Ga}_{2} \mathrm{O}_{3}\right.$ based), the zero dispersion wavelength, $\lambda_{0}$, typically falls between 2 and $3 \mu \mathrm{m}$. The material dispersion of such glasses at $1.55 \mu \mathrm{m}$ is therefore always large and normal. An optical fiber with a flat and near-zero dispersion profile in the 'extended' telecommunication window (1.3-1.7 $\mu \mathrm{m})$ requires a significant amount of waveguide dispersion to compensate for the material dispersion, which can only be achieved with a high index contrast between the fiber core and the cladding.

Table 1 Summary of $\lambda_{0}$ of various types of optical glass

\begin{tabular}{|c|c|c|c|c|c|}
\hline & Glass & $\lambda_{1}$ & $\lambda_{0}$ & $\lambda_{2}$ & References \\
\hline \multirow{2}{*}{$\begin{array}{c}\text { Low refractive } \\
\text { index } \\
(\mathrm{n}<1.7) \\
\end{array}$} & Silica & $<\sim 0.2 \mu \mathrm{m}$ & $1.27 \mu \mathrm{m}$ & $<5 \mu \mathrm{m}$ & [12] \\
\hline & Fluoride & $<\sim 0.2 \mu \mathrm{m}$ & $1.5-1.7 \mu \mathrm{m}$ & $5-10 \mu \mathrm{m}$ & [13] \\
\hline \multirow{3}{*}{$\begin{array}{l}\text { High refractive } \\
\text { index }(n>1.7)\end{array}$} & HMO-silicate & $0.3-0.4 \mu \mathrm{m}$ & $\sim 2 \mu \mathrm{m}$ & $<5 \mu \mathrm{m}$ & [14] \\
\hline & $\begin{array}{l}\text { HMO non- } \\
\text { silicate glass: } \\
\text { e.g., tellurite, } \\
\text { germanate, } \\
\text { gallate }\end{array}$ & $0.3-0.7 \mu \mathrm{m}$ & $2-3 \mu \mathrm{m}$ & $5-10 \mu \mathrm{m}$ & {$[15,16]$} \\
\hline & Chalcogenide & $0.6-0.9 \mu \mathrm{m}$ & $5-7 \mu \mathrm{m}$ & $>10 \mu \mathrm{m}$ & [13] \\
\hline
\end{tabular}

\subsection{Properties of selected commercial high-index lead silicate glasses}

In this work we focus on lead-silicate glasses, which represent an attractive family of glasses due to their superior thermal and crystallization stability and less steep viscosity-temperature characteristic curves as compared to most compound glass alternatives [17]. In particular, we employ three commercially available lead silicate glasses, Schott SF57, SF6, and LLF1, to fabricate various HNLFs with tailored dispersion at telecommunication wavelengths. Table 2 shows the basic thermal and optical properties of these three glasses. In particular, it can be seen that SF57 and SF6 glasses have high nonlinear indices $\mathrm{n}_{2}$ of $41 \times 10^{-20}$ and $22 \times 10^{-20} \mathrm{~m}^{2} / \mathrm{W}$, respectively.

Table 2 Summary of basic physical properties of SF57, SF6 and LLF1 glasses [14, 18, 19].

\begin{tabular}{|l|l|l|l|l|l|}
\hline & $\begin{array}{c}\text { Glass transition } \\
\text { temperature, } \mathrm{T}_{\mathrm{g}} \\
\left({ }^{\circ} \mathrm{C}\right)\end{array}$ & $\begin{array}{c}\text { Softening Point, } \mathrm{T}_{10}{ }^{7.6} \\
\left({ }^{\circ} \mathrm{C}\right)\end{array}$ & $\begin{array}{c}\lambda_{0} \\
(\mu \mathrm{m})\end{array}$ & $\begin{array}{c}\text { Refractive } \\
\text { index } \mathrm{n} \\
(\text { at } 1.55 \mu \mathrm{m})\end{array}$ & $\begin{array}{c}\text { Nonlinear } \\
\text { refractive } \\
\text { index } \mathrm{n}_{2} \\
\left(\mathrm{~m}^{2} / \mathrm{W}\right)\end{array}$ \\
\hline Schott SF57 & 414 & 519 & 1.97 & 1.80 & $41 \times 10^{-20}$ \\
\hline Schott SF6 & 423 & 538 & 1.93 & 1.76 & $22 \times 10^{-20}$ \\
\hline Schott LLF1 & 415 & 628 & 1.54 & 1.53 & $6 \times 10^{-20}$ \\
\hline
\end{tabular}

The glass viscosity is paramount for every thermal engineering step involved in the fiber fabrication, e.g. preform fabrication and/or fiber drawing. Fig.5 shows the viscosity curves of Schott SF57, LLF1, and SF6 glasses measured using the parallel plate method [6]. Note that the temperature corresponding to the viscosity of $10^{7.6}$ poise, $\mathrm{T}_{10}{ }^{7.6}$ (shown in Table 2), is the glass softening temperature, around which glass extrusion can be performed. As will be demonstrated in the following, despite the large difference in viscosity between these glasses at any given temperature, we have consistently been able to draw different combinations of them into fibers. 


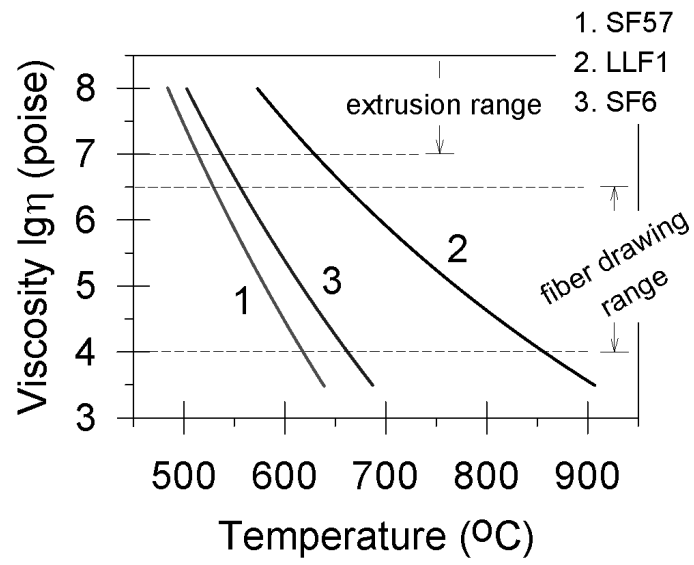

Fig. 5. Measured viscosity curves of Schott SF57, LLF1, and SF6 glasses.

\section{Fiber design, fabrication, and characterization}

In this section we review our recent progress in developing highly nonlinear lead-silicate glass fibers, including holey fibers, all-solid 1D MOFs, and W-type index profile fibers, with flattened and near-zero dispersion profiles around $1.55 \mu \mathrm{m}$.

\subsection{Air-suspended core holey fiber}

A holey fiber is a single-material optical fiber where light guidance is obtained through an array of longitudinal air holes surrounding a solid glass core. Holey fiber technology can achieve high nonlinearity and tailored fiber dispersion by exploiting the large index contrast between glass and air and by engineering wavelength-scale microstructures in the fiber cladding. In particular, by suitably designing the size, shape and spacing of the air holes, the waveguide dispersion and consequently the total dispersion of the fiber can be significantly modified.

Air-suspended-core (ASC) HFs [20-24], with a small glass core that is effectively suspended in air, represent the simplest holey fiber design. Fig.6(a) shows the cross-section of a typical ASC HF comprising a small glass core supported by three long and thin spokes. The core diameter of an ASC HF, $d_{c o r e}$, is defined as the diameter of the circle enclosed within the outer triangular core region; $t_{s p o k e}$ is the thickness of the spokes, and $l_{\text {spoke }}$ is the length of the spokes. Because the supporting spokes are much smaller in thickness and much longer in length as compared to the operational wavelength, such a HF can almost be regarded as an optical fiber with a solid glass core suspended in an air cladding. This fiber structure provides the largest possible air-filling fraction in the cladding, and therefore the largest index contrast between the core and the cladding. Hence, this particular fiber design achieves the highest numerical aperture

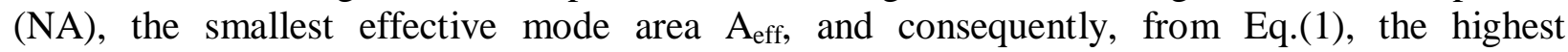
nonlinearity $\gamma$ [24-27]. Moreover, due to the combination of the largest possible index-contrast with a sub-wavelength-sized core diameter, the strongest waveguide dispersion can be obtained in an ASC HF, thus allowing the shortest possible zero dispersion wavelength (ZDW), which can be as far as several hundred nanometers $\left(\sim 0.5 \lambda_{0}\right)$ away from the material ZDW $\lambda_{0}$. 


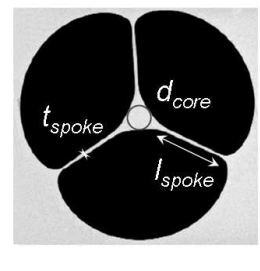

(a)

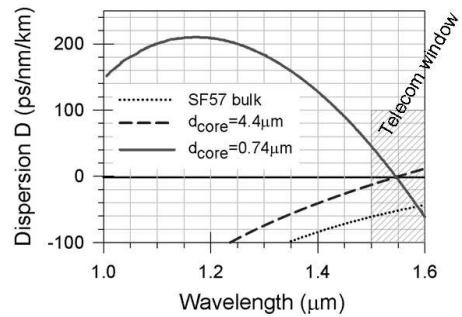

(b)

Fig. 6. (a) Cross-section of a typical ASC HF. (b) Calculated dispersion curves of SF57 ASC HFs with ZDW at $1.55 \mu \mathrm{m}$.

Commercial high lead-content silicate Schott SF57 glass $\left(\mathrm{SiO}_{2}-\mathrm{PbO}, \mathrm{PbO}>40 \mathrm{~mol} . \%\right)$, was selected for the fabrication of a single-material ASC-HF with ZDW at $1.55 \mu \mathrm{m}$. As shown in the simulation results of Fig.6(b), in order to obtain a ZDW of $1.55 \mu \mathrm{m}$, the core diameter $\mathrm{d}_{\text {core }}$ of the ASC HF should be either $4.4 \mu \mathrm{m}$ or $0.74 \mu \mathrm{m}$. However, the former HF is a highly multimoded fiber, which is undesirable for most applications and in particular for all-optical processing applications of telecommunication signals. In contrast, the smaller core structure achieves single mode guidance in addition to a shifted zero-dispersion wavelength within the telecom C-band $(1.53$ to $1.565 \mu \mathrm{m})$ and an extremely high nonlinearity $\gamma$, calculated to be around $2000 \mathrm{~W}^{-1} \mathrm{~km}^{-1}$ at $1.55 \mu \mathrm{m}$. As will be shown in the following, however, although fabrication of ASC HF with submicron core dimensions is possible, the precise position of the ZDW is extremely sensitive to the core diameter, thus posing very stringent constraints in terms of consistency of the core diameter along the fiber length, which are very challenging to meet with the current fabrication techniques.

The following procedure is generally employed in order to fabricate a non-silica ASC HF with a submicron core diameter: (1) a structured preform is extruded from a glass billet; (2) the structured perform is elongated or drawn into a $\sim \mathrm{mm}$ sized cane; and (3) the cane is inserted into a jacketing tube and this preform assembly is then drawn into a fiber with the targeted core dimension through a method very similar to the rod-in-tube technique [28]. A schematic of the fabrication technique for the extrusion of structured non-silica glass preforms is illustrated in Fig.7(a). First the glass billet is heated to above the glass softening temperature $\left(519^{\circ} \mathrm{C}\right.$ for SF57). Then pressure is applied through the ram onto the glass, thereby forcing a viscous glass flow through the structured die. The structured preform is then cooled down to room temperature.

Fig.7(b) shows an optical photograph of the cross-section of the extruded 10mm outer diameter (OD) SF57 preform. A Scanning Electron Microscopy (SEM) image, Fig.7(b) and (c), shows that the structured preform has a glass core with a diameter of $130 \mu \mathrm{m}$, and three high aspect ratio supporting spokes, about $1.45 \mathrm{~mm}$ in length and only $7 \mu \mathrm{m}$ thick.

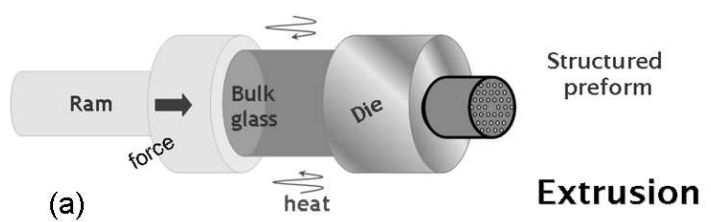

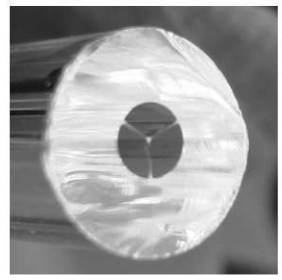

(b)

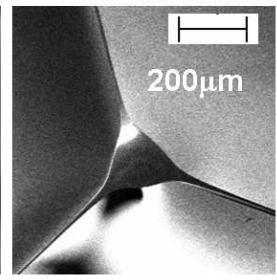

(c) 
Fig. 7. (a) Schematic of extruding a soft glass structured preform. (b) Optical photograph and (c) SEM photograph of an extruded SF57 preform with $130 \mu \mathrm{m}$ core diameter and 10mm OD.
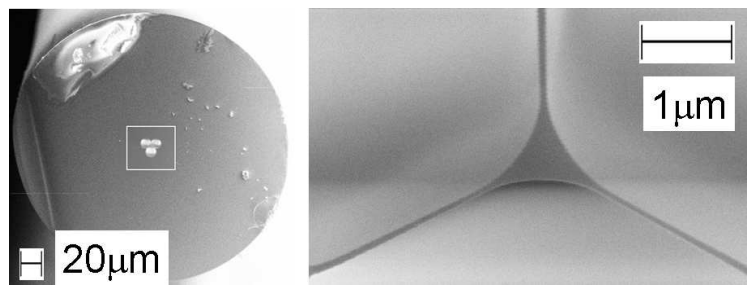

Fig. 8. SEM photographs of SF57 ASC HF with $d_{\text {core }}$ of $0.74 \mu \mathrm{m}$

The preform was then elongated into a small cane and inserted into a jacketing tube with suitable diameter. The ASC HF with a submicron diameter core was then drawn. Negative pressure was used during the fiber drawing in order to eliminate the gap between the structured cane and the jacket tube and avoid the presence of any interstitial holes. Fig.8 shows SEM images of the HF with a core diameter $\mathrm{d}_{\text {core }}=0.74 \mu \mathrm{m}$. The average thickness $\mathrm{t}_{\text {spoke }}$ and length $1_{\text {spoke }}$ of the three spokes are $0.12 \mu \mathrm{m}$ and $4.0 \mu \mathrm{m}$, respectively.

Based on the fiber geometry obtained via high resolution SEM images, the wavelength dependent dispersion curve was calculated using full vector Finite Element Method (FEM) simulations [29]; the results are shown in Fig. 9. The ASC HF has a ZDW at $1.59 \mu \mathrm{m}$ and a dispersion slope of $0.7 \mathrm{ps} / \mathrm{nm}^{2} / \mathrm{km}$ at $1.55 \mu \mathrm{m}$. The nonlinearity $\gamma$ of the fiber is calculated to be $1850 \mathrm{~W}^{-1} \mathrm{~km}^{-1}$ at $1.55 \mu \mathrm{m}$, close to the maximum theoretical value for SF57 HFs [23]. However, Fig.9 also confirms that the dispersion of this fiber design is extremely sensitive to the core diameter $\mathrm{d}_{\text {core }}$, which is the primary parameter used to control the dispersion profile in an ASC $\mathrm{HF}$. By reducing $\mathrm{d}_{\text {core }}$ by approximately $5 \%$, from $0.74 \mu \mathrm{m}$ down to $0.70 \mu \mathrm{m}$, the $\mathrm{ZDW}$ of the HF shifts by over $100 \mathrm{~nm}$ from $1.55 \mu \mathrm{m}$ to $1.45 \mu \mathrm{m}$. This observation suggests that, in order to obtain a ZDW within the C-band, the core diameter needs to be controlled with an accuracy better than $10 \mathrm{~nm}$.

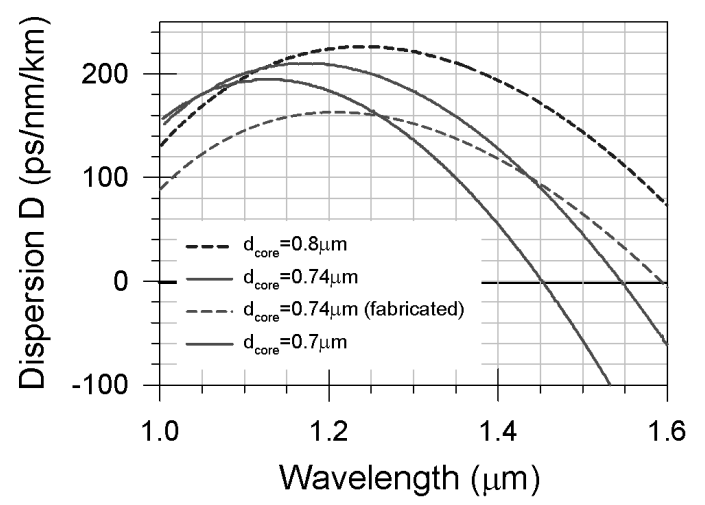

Fig. 9. Calculated dispersion curves of the fabricated SF57 glass ASC HF and the calculated dispersion curves of idealized fibers with $\mathrm{d}_{\text {core }}$ of $0.70,0.74$ and $0.80 \mu \mathrm{m}$.

It is worth noting that a significant difference is observed in Fig. 9 between the dispersion profiles calculated for an idealized ASC HF structure (see Fig.6) and the actual fabricated structure with the same core diameter $\mathrm{d}_{\text {core }}$ of $0.74 \mu \mathrm{m}$ (Fig.8). This discrepancy is due to the fact that the two structures have slightly different core geometries outside the enclosed circle (Fig.6(a)) which arise as a consequence of the surface tension on the submicron core during the fiber drawing. For ASC HFs with a submicron-diameter core, the guided mode will inevitably expand beyond the glass core in the air holes and in the transition area with the glass spokes. Thus the dispersion of the real fiber is not only sensitive to the core diameter but also to the 
geometry of the transition area between the core and the supporting spokes. This makes it very difficult to precisely control the dispersion profile, e.g., the location of the ZDW, of the submicron ASC HF. This difficulty, in spite of its extremely high nonlinearity, has to date limited the practical use of this fiber type.

\subsection{Holey fiber with complex microstructured cladding}

A key requirement for several applications of HNLFs based on four-wave-mixing (FWM) is that in order to satisfy exactly or approximately the phase matching condition over a broad wavelength range the fibers should have both zero dispersion and zero dispersion slope at the required wavelength of operation, typically $1.55 \mu \mathrm{m}$. [30]. In the case of applications requiring short (i.e., meter or sub-meter) device lengths, these conditions can be somewhat relaxed and near-zero dispersion and dispersion slope at $1.55 \mu \mathrm{m}$ are acceptable.

The air-suspended structure described in the previous section does not allow easy control of both dispersion and dispersion slope independently, thus a different and more complex HF structure is required [31,32] where additional degrees of freedom are available in engineering these properties. We have therefore considered an omitted centre-hole structure based on a triangular lattice. Fig. 10 shows a schematic of the fiber structure and an "optical property map" displaying how the chromatic dispersion and the dispersion slope depend on the structural parameters in the case of a fiber made of SF57 glass at $1.55 \mu \mathrm{m}$. The condition for zero dispersion and zero dispersion slope is highlighted in the graph and corresponds to a hole-to-hole spacing $(\Lambda)$ of $1.36 \mu \mathrm{m}$ and a relative hole size $(\mathrm{d} / \Lambda)$ of 0.454 . The fiber nonlinearity $\gamma$ of this particular fiber is predicted to be $470 \mathrm{~W}^{-1} \mathrm{~km}^{-1}$ at $1.55 \mu \mathrm{m}$. By comparison, a silica highly nonlinear HF with flattened and near-zero dispersion at $1.55 \mu \mathrm{m}$ only has $\gamma \sim 10-20 \mathrm{~W}^{-1} \mathrm{~km}^{-1}[32$, 33].

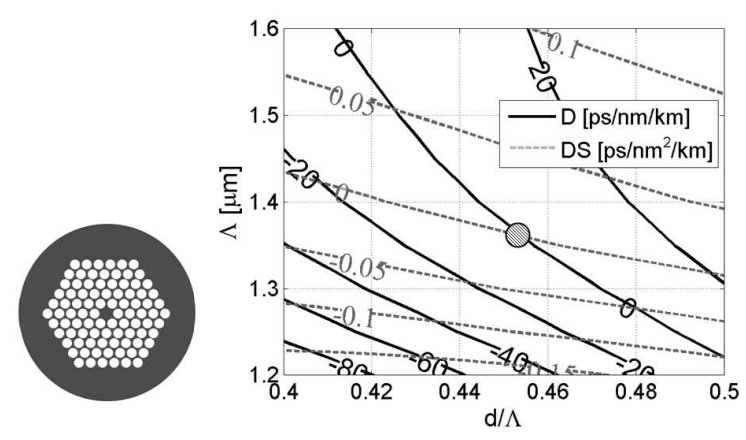

Fig. 10. Schematic of a HF based on a triangular lattice of holes and a omitted centre-hole structure and optical property map (at $1.55 \mu \mathrm{m}$ ) for a SF57 HF. Dispersion (D) contours (in ps/nm/ $\mathrm{km}$ ) are shown as solid curves, while dispersion slope (DS) contours (in $\mathrm{ps} / \mathrm{nm}^{2} / \mathrm{km}$ ) are shown in dashed curves. The gray circle represents the cross point of the zero dispersion and dispersion slope.

The structure shown in Fig.10 has 5 rings of cladding holes, and this is required in order to achieve a low confinement loss of the fundamental mode $\mathrm{LP}_{01}$. Even though reports show that preform extrusion should still be possible for the fabrication of such a structure [34], extruding preforms for structures with a large number of moderately sized holes $(\mathrm{d} / \Lambda \approx 0.45)$ is considered to be quite challenging, especially when the precise size and overall shape of the structure is of primary importance. However, the numerical simulation indicates that, (1) the dispersion of the final holey fiber is mainly determined by the hole dimensions of the first and second ring of holes around the solid core, consequently (2) a HF with slightly graded hole sizes will still provide similar flattening and a near-zero dispersion profile as a HF with all identical holes would. Therefore we have investigated an alternative method, based on the Structured Element Stacking Technique (SEST) [31,35]. First, a preform with three rings of holes was obtained by 
extrusion (note that the complexity in extruding this structure is significantly lower than the corresponding of the 5-ring structure of Fig.10). An optical microscope image of its cross section is shown in Fig.11(a); the corner-corner length of the preform is $16.2 \mathrm{~mm}$. It is important to notice that, in order to pre-compensate for the non-uniform expansion of the holes that occurs during the subsequent fiber drawing step, the hole-to-spacing ratio $\mathrm{d} / \Lambda$ of the different rings of holes needs to be graded.

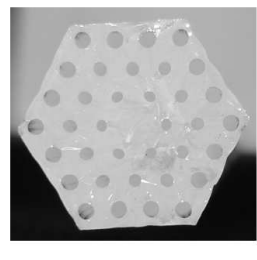

(a)

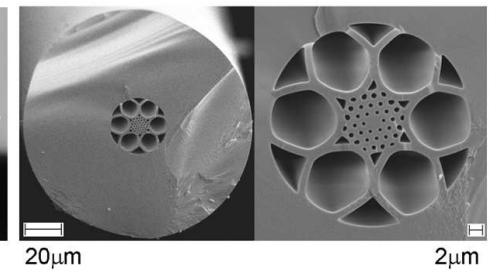

(b)

Fig. 11. (a) Optical microscope image of an extruded SF57 preform with 3 rings of holes surrounding a solid core. (b) SEM images of the final double clad HF; the central structure is surrounded by a further ring of expanded air holes.

The extruded stacked preform was then elongated to a cane and stacked at the centre of six other thin SF57 capillaries inside an SF57 jacket tube. This assembled preform was then drawn into a fiber. Fig.11(b) shows SEM images of the fabricated HF, where the two different holey claddings can clearly be identified. The first cladding, immediately surrounding the core, has an average hole spacing $\left(\Lambda_{1}\right)$ of $1.60 \mu \mathrm{m}$; the hole diameter $\mathrm{d}_{1}$ of the first ring of holes is $0.56 \mu \mathrm{m}$, and $\mathrm{d} / \Lambda$ varies from 0.35 to 0.5 in the three rings composing this inner cladding. The outer cladding, consisting of six large holes, exhibits a large hole-to-spacing ratio $d_{2} / \Lambda_{2}=0.85$. The dispersion of this HF is primarily determined by the structural parameters of the inner first cladding, while the confinement loss of the $\mathrm{LP}_{01}$ mode is mainly determined by the structure of the outer cladding. Although numerical simulations indicate that this HF theoretically supports a few modes, effective single-mode guidance was experimentally observed at $1.55 \mu \mathrm{m}$ as confirmed by analysis of near field modal profiles. We speculate that this is due to a combination of a good launch into the fundamental mode and higher differential loss of the higher order modes. The $\gamma$ value of this HF was measured as $270 \mathrm{~W}^{-1} \mathrm{~km}^{-1}$ at $1.55 \mu \mathrm{m}$, using the Boskovic method [36], whereas the propagation loss was measured to be $3.0 \pm 0.1 \mathrm{~dB} / \mathrm{m}$ at $1.55 \mu \mathrm{m}$.

We obtained an accurate estimate of the fiber dispersion through numerical calculations based on the actual fiber structure obtained by high resolution SEM images; the result is shown in Fig.12. Measurements at $1.55 \mu \mathrm{m}$ wavelength have confirmed a dispersion value of $-17 \mathrm{ps} / \mathrm{nm} / \mathrm{km}$ (indicated by a circle in Fig. 12) and a dispersion slope of $0.10 \mathrm{ps} / \mathrm{nm}^{2} / \mathrm{km}$. Fig. 12 also shows an optimized dispersion profile which exhibits minimal dispersion across the wavelength range $1500-1600 \mathrm{~nm}$. A fiber with these characteristics would be ideal for applications targeting parametric processes. In order to obtain this profile, it would be necessary to (1) reduce the hole spacing in the first cladding, $\Lambda_{1}$, from $1.60 \mu \mathrm{m}$ to $1.36 \mu \mathrm{m}$ and (2) increase the hole-to-spacing ratio in the first cladding, $\mathrm{d}_{1} / \Lambda_{1}$, from 0.35 to 0.45 . The second point is particularly challenging to achieve in practice. First, one has to carefully choose different values of $d / \Lambda$ for each ring of the extruded preform, in order to compensate for the distortion of the holey microstructure during fiber drawing, where the different rings of holes generally experience different expansion. Moreover, the actual amount of hole expansion is strongly dependent on the detailed fiber drawing conditions, such as length/shape of the hot-zone of the furnace, diameter of the preform and preform feed rate, making it very hard to obtain a consistent structure. We therefore concluded that, for this HF structure, it is currently very difficult to obtain the target parameters required in order to achieve near-zero chromatic dispersion and dispersion slope at $1.55 \mu \mathrm{m}$. It is however possible that continued improvements of the extrusion and fiber drawing processes may lead to more consistent and usable fibers. 


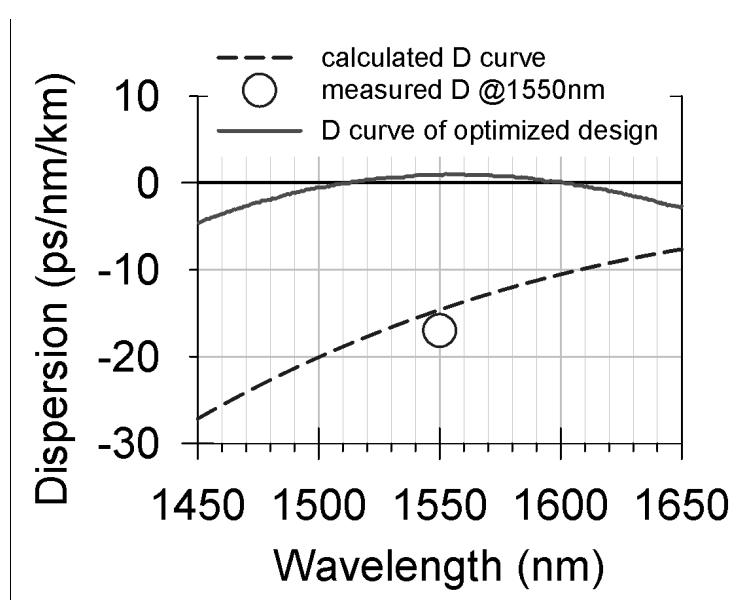

Fig. 12. Calculated dispersion curve of the optimum SF57 HF design $(\mathrm{d} / \Lambda=0.454, \Lambda=1.36 \mu \mathrm{m}$, solid line), calculated dispersion curve of the fabricated SF57 HF with complex holey cladding (dashed line) and measured dispersion at $1.550 \mu \mathrm{m}$ (circle).

\subsection{All-solid 1D microstructured fiber}

We have seen in the previous sections that, when targeting a flattened and near-zero chromatic dispersion at the $\mathrm{ps} / \mathrm{nm} / \mathrm{km}$ level at about $1.55 \mu \mathrm{m}$ in holey fiber structures, it is necessary to achieve control of hole size and spacing to the sub- $\mu \mathrm{m}$ level. This leads to two fabrication-related problems: (1) sub- $\mu \mathrm{m}$ control of hole size is very difficult to reliably achieve in practice because of the complex interdependence between temperature, surface tension and internal pressure in the holes. This will almost inevitably cause considerable deviations between the initial structure, e.g. in an extruded preform or cane, and the final fiber, which will in turn cause a significant deviation from the targeted dispersion profile; (2) for hole dimensions below the wavelength scale, the surface roughness of the glass at the holes, in conjunction with the high index contrast between air and glass, will generally result in a high scattering loss at the air/glass interface [4, 24].

An elegant solution to overcome these drawbacks is to use a high index-contrast all-solid microstructured optical fiber (MOF) rather than a holey fiber: in these microstructured fibers holes are replaced with solid regions of a second glass, which significantly reduces the occurrence of structural deformations induced during the fiber drawing and helps maintain the original relative scale factor of high index/low index features when going from preform to fiber [37-39]. Furthermore, any losses due to interface scattering can be substantially reduced in this case by accurately polishing the surfaces of the glass elements used to fabricate the preform. Following this idea, we recently fabricated an all-solid one-dimensional (1D) MOF with low loss, high nonlinearity and low dispersion at $1.55 \mu \mathrm{m}$ [39]. Two chemically compatible commercial optical glasses, SF6 and LLF1 (respectively high-index and low-index, see Table 2), were used for this MOF. As shown in Fig.5, there is a substantial thermal mismatch in the viscosities of the two glasses. Although such mismatch affects in principle both preform extrusion (carried out at viscosity ranges of $10^{9}-10^{7}$ poise) and fiber drawing $\left(10^{6.5}-10^{4}\right.$ poise) processes, we found that the two glasses are in fact thermally compatible and that it was possible to produce high optical quality preforms and fibers. We pursued a fiber design comprising a number of alternating high- and low-index coaxial rings with the layer thickness ultimately determining the optical properties of the fiber. The structured preform was fabricated by coextruding alternately stacked high- and low-index glass discs through a circular aperture (see Fig.13). Note that all the glass discs used within our extrusion process were of high optical quality with accurately and finely polished surfaces. The co-extrusion method employed to form 
the multiple coaxial ring structure in the extruded preform is explained in more detail in refs. $[38,39]$.

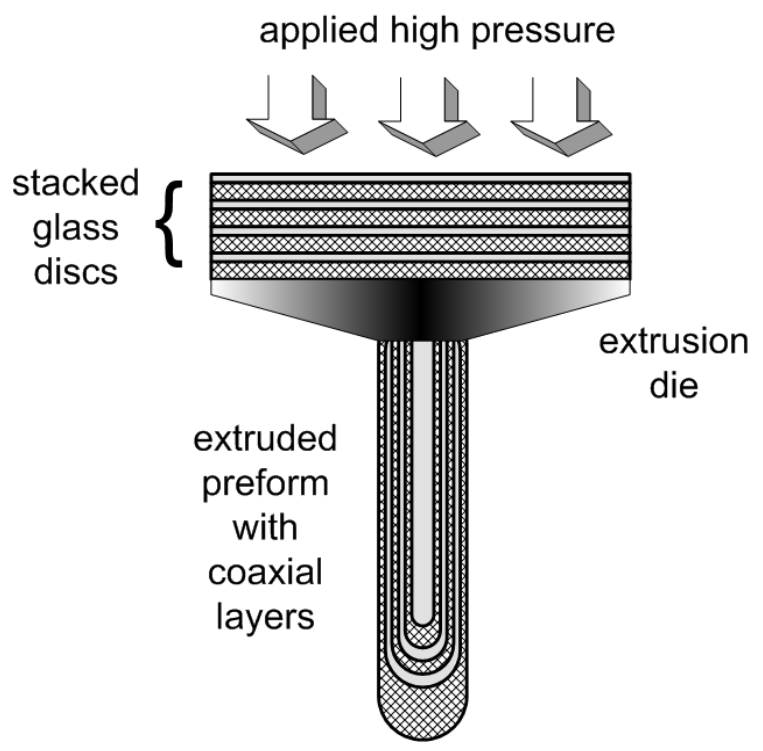

Fig. 13. Schematic of the fabrication of a preform with coaxial-ring structures by coextrusion of alternately stacked high- and low-index glass discs.

A structured preform with an outer diameter (OD) of $10 \mathrm{~mm}$ was elongated to a cane of $1.09 \mathrm{~mm}$ diameter and then inserted into an extruded SF6 glass jacket tube with an OD of $15.80 \mathrm{~mm}$ and an inner diameter (ID) of $1.10 \mathrm{~mm}$. From a single fiber draw, fibers with core diameters of 5.0, 4.1,3.7, and $3.3 \mu \mathrm{m}$ have been fabricated with excellent yield of over $100 \mathrm{~m}$ in length. Fig.14(a) shows SEM images of the obtained MOF, where the regions of different glass are highlighted via a z-contrast technique: a high index glass has a higher brightness than a lowindex glass, as explained elsewhere [37]. The fiber shown has a $150 \mu \mathrm{m}$ OD and a high-index SF6 core of $3.7 \mu \mathrm{m}$ in diameter. The core is surrounded by alternating LLF1 and SF6 glass rings. The three low-index rings have thicknesses of $1.1,0.6$, and $0.6 \mu \mathrm{m}$, respectively. The two highindex rings have thicknesses of 0.4 and $0.3 \mu \mathrm{m}$, respectively. Fig.14(b) illustrates the profile of the refractive index of the 1D MOF, according to the structural parameters obtained from high resolution SEM images.

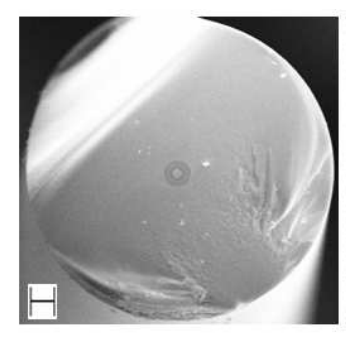

$10 \mu \mathrm{m}$

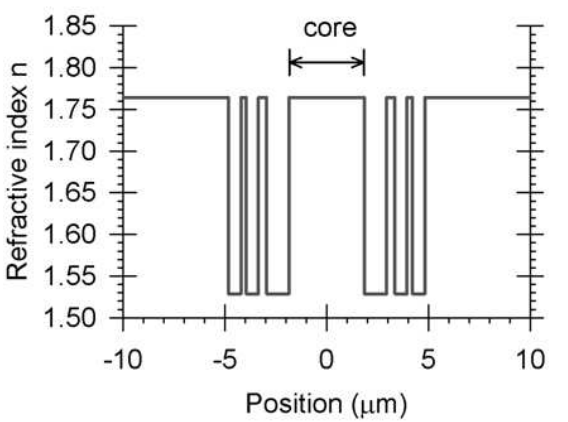

(a)

(b) 
Fig. 14. (a) SEM images and (b) index profile of the solid MOF with $3.7 \mu \mathrm{m}$ core diameter.

Fig.15 compares the fiber structure to that of the $1.09 \mathrm{~mm}$ OD cane from which it was fabricated. As a visual aid, four rectangular frames between the cane and the fiber are also superimposed on the images to mark the various boundaries of the two glasses. Note that on the cane a third high-index ring is present, which becomes merged with the outer jacketing glass tube during the fiber draw. It is very obvious that the shape and relative size of the high/low index features in the cane and in the final fiber are completely identical, even though the features in the final fiber are micron-scale. This clearly demonstrates the advantages of using all-solid MOFs over air-filled HFs. The former can give far more predictable and reliable control of the microstructure during the various fabrication stages and right up to the final fiber. This is critically important when targeting MOFs with strongly structure-dependent fiber properties.

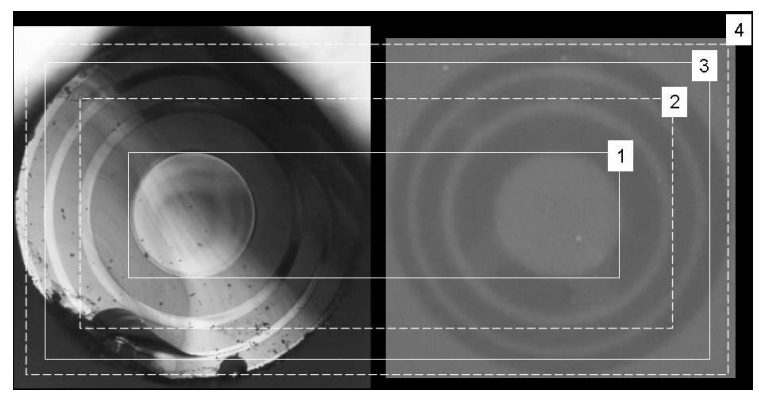

Fig. 15. Comparison between the structural parameters of the cane (left) and the final fiber (right) for the all-solid MOF with coaxial-ring structure.

We calculated the optical properties of the solid MOF assuming an idealized structure with parameters matching those measured from high resolution SEM images. It was found that the MOF shown in Fig.14 and 15 (3.7 $\mu \mathrm{m}$ core diameter) supports a few higher-order modes (HOMs) at $1.55 \mu \mathrm{m}$. The confinement loss of all HOMs is however of several tens of $\mathrm{dB} / \mathrm{m}$ and effectively single-mode guidance was observed from this 1D MOF over meter-scale lengths (see near-field mode profile in Fig.16(a)). Fig.16(a) also shows the intensity profile of the simulated fundamental $\mathrm{LP}_{01}$ mode. Using the cutback method, the propagation loss of the fundamental mode was measured as $0.8 \pm 0.2 \mathrm{~dB} / \mathrm{m}$ at $1.55 \mu \mathrm{m}$; for increased accuracy, the loss value was determined with multiple cutbacks (the total cutback length of $2.4 \mathrm{~m}$ ) (see Fig.16(b)). This measured loss figure is very close to the bulk attenuation deduced from the published data of commercial Schott SF6 glass [14]. To the best of our knowledge, this loss figure is one of the lowest ever reported in a non-silica glass MOF. We attribute such a very low loss to the following reasons: (1) the starting glass discs used for preform extrusion were finely polished to ensure a high optical quality and (2) the extrusion was optimized in terms of temperature and speed to minimize the formation of any bulk and interface defects in the structured preform. By contrast, conventional soft-glass HF fabrication procedures involving extrusion [18] or drilling $[4,34]$, tend to produce significant surface roughness and/or surface defects in the preforms, which inevitably impact the quality of the air/glass interfaces in the final fibers and can give rise to relatively high attenuation due to light scattering. 


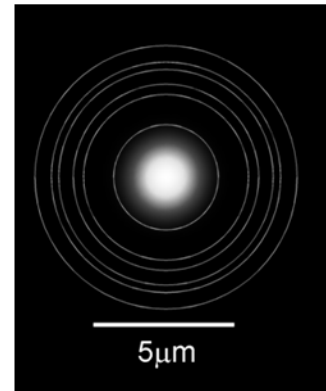

Simulated $L P_{01}$ mode

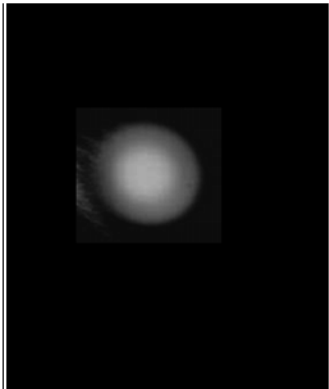

Observed $\mathrm{LP} \mathrm{P}_{01}$ mode

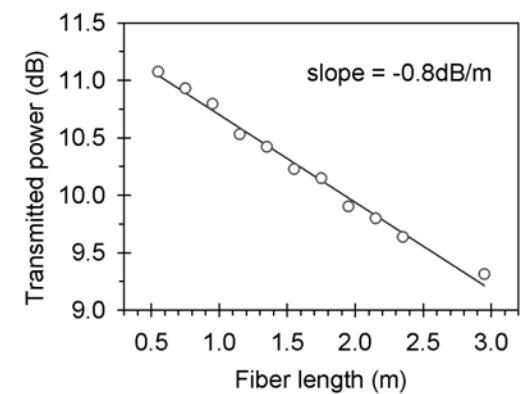

(a)

(b)

Fig. 16. (a) Simulated (left) and observed (right) $\mathrm{LP}_{01}$ mode of $1 \mathrm{D} \mathrm{MOF}$; (b) power transmission versus fiber length obtained from a cutback measurement, and linear fit.

The effective nonlinearity $\gamma$ of the 1D MOF was again measured using the Boskovic method [36], which gave a value of $120 \mathrm{~W}^{-1} \mathrm{~km}^{-1}$ at $1.55 \mu \mathrm{m}$. Based on the numerical modeling, the effective mode area $\mathrm{A}_{\text {eff }}$ was calculated to be $6.7 \mu \mathrm{m}^{2}$ at $1.55 \mu \mathrm{m}$, which corresponds (using the nonlinear refractive indices $\mathrm{n}_{2}$ of the two glasses) to a nonlinear coefficient $\gamma$ of $130 \mathrm{~W}^{-1} \mathrm{~km}^{-1}$, in good agreement with the measured value.

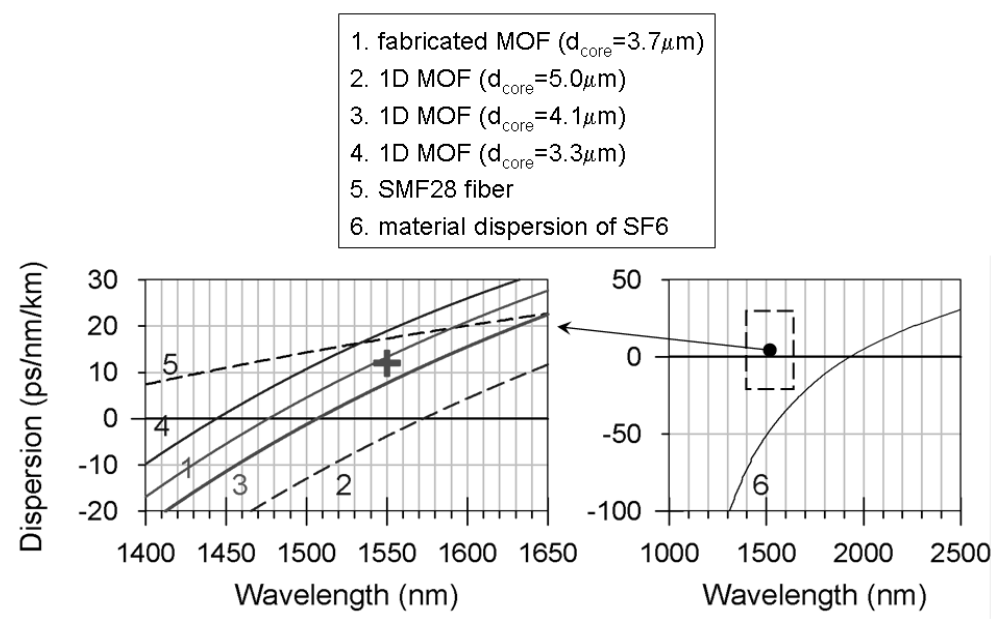

Fig. 17. Calculated dispersion curve and measured dispersion (marked by the cross at $1.55 \mu \mathrm{m}$ ) of the 1D MOF with core diameter $d_{\text {core }}=3.7 \mu \mathrm{m}$, dispersion curves of the fabricated 1D MOFs with $\mathrm{d}_{\text {core }}=5.0,4.1$ and $3.3 \mu \mathrm{m}$, as well as dispersion of silica SMF28 fiber and of bulk SF6. Note that the left figure is corresponding to the rectangular inset frame in the right figure.

The dispersion of the 1D MOF was measured to be $+12.5 \mathrm{ps} / \mathrm{nm} / \mathrm{km}$ at $1.55 \mu \mathrm{m}$ using the FWM method [40] - this value is shown by a cross in Fig.17. The chromatic dispersion was again determined numerically using the structural parameters of the real fiber as input; we employed a full vector finite-element (FEM) method and independently confirmed the results via a semi-analytic transfer matrix approach. The dispersion curve shown in Fig. 17 indicates that this $1 \mathrm{D}$ MOF is in practice a dispersion-shifted fiber with a ZDW at about $1.475 \mu \mathrm{m}$ and a 
dispersion slope of $0.16 \mathrm{ps} / \mathrm{nm}^{2} / \mathrm{km}$ at $1.55 \mu \mathrm{m}$. The calculated dispersion at $1.55 \mu \mathrm{m}$ is in good agreement with the measured value. For reference, the dispersion curve of commercial silica SMF28 fiber is also illustrated in Fig. 17. At $1.55 \mu \mathrm{m}$ the dispersion value of the MOF is close to that of SMF28, even though the effective nonlinearity of the MOF is 120 times higher than the SMF28. A few meters of this 1D MOF can therefore exhibit a total nonlinearity equal to that of several hundreds of meters of SMF28 whilst exhibiting much less net dispersion - an important property for many nonlinear applications such as FWM. Fig.17 also illustrates the material dispersion of bulk SF6 glass and the calculated dispersion curves of the 1D MOFs with core diameters of $5.0 \mu \mathrm{m}, 4.1 \mu \mathrm{m}$, and $3.3 \mu \mathrm{m}$ fabricated from the same cane. It can be seen that reducing the size of the wavelength-scale features in the microstructured cladding generates strong and positive waveguide dispersion, i.e. opposite in sign to the material dispersion of the core glass, thus shifting the ZDW of the fiber to shorter wavelengths. For instance, the ZDW shifts from $1.57 \mu \mathrm{m}$ to $1.44 \mu \mathrm{m}$ when the core diameter is decreased from $5.0 \mu \mathrm{m}$ to $3.3 \mu \mathrm{m}$.

The characterization results suggest that the $1 \mathrm{D}$ MOF is suitable for applications relying on FWM in the $1.55 \mu \mathrm{m}$ band. To investigate this further we set up a FWM-based wavelength conversion scheme, in which a cw wave and a pulsed data signal (which acted as the pump) were launched into a $1.5-\mathrm{m}$ long piece of the $1 \mathrm{D}$ MOF. Broadband wavelength conversion with a $-3 \mathrm{~dB}$ bandwidth of $17 \mathrm{~nm}$ was observed, covering a substantial part of the C-band [36]. Note however, that a short length of fiber was used in order to achieve a broad conversion bandwidth. This in practice limited the conversion gain achieved and did not fully utilize the benefits of the exceptionally low loss exhibited in this fiber. Further optimization of the dispersion properties is required in order to improve the performance of the fiber when considered for broadband applications involving parametric effects. In order to further flatten the dispersion of these fibers, a more accurate control of the ratio between the core diameter and the thickness of the innermost rings would be required. Although the all solid nature of these fibers allows to achieve a remarkably low transmission loss (for a soft glass MOF) and excellent structural consistency when drawing from preform to cane and finally into fiber, it is still very challenging to control the thickness of many rings in the preform independently using the co-extrusion method described above. Therefore we considered a further and simpler fabrication approach, as described below.

\subsection{W-type step-index profile fiber with high index contrast}

We looked for a fiber design and a fabrication approach which would enable us to maintain the desirable deformation-free properties of all-solid fibers, while allowing a better control of the overall structural features and dispersion properties. In an attempt to simplify the design, we went back to the most basic optical fiber design possible, i.e. the step-index design. Waveguide theory shows that, for any given core material, it is always possible, in principle, to engineer a step-index fiber (with diameter $d$ and step index $\Delta n$ ) such that, at a given wavelength, the waveguide dispersion $\left(\mathrm{D}_{\mathrm{w}}\right)$ exactly compensates for the material dispersion $\left(\mathrm{D}_{\mathrm{m}}\right)$ in absolute value and slope, thus creating an overall dispersion flattened profile [41]. Modifying $\Delta n$ can be regarded as a 'material'-based route to control the fiber dispersion, as opposed to the 'structure' based approach followed in HFs where the hole sizes are modified. Although the 'structure'based route has the advantage of requiring only one glass, as already discussed in the previous sections, there are inherent difficulties in engineering the desired air hole dimensions with the required precision.

In this work, we chose SF57 glass as the core material and systematically searched for a suitable, compatible cladding glass generating a near-zero dispersion profile around $1.55 \mu \mathrm{m}$ in a step-index fiber structure. Combining practical material constraints with the results from numerical simulations, we found that Schott LLF1 glass is an excellent option as a cladding medium [42]. When the core diameter is reduced to wavelength-scale dimensions, the SF57- 
LLF1 combination provides a high enough index-contrast and hence a strong enough waveguide dispersion to compensate for the core material dispersion. As shown in Fig.18(a), such a stepindex fiber with a core diameter $\mathrm{d}_{0}$ of $1.64-1.68 \mu \mathrm{m}$ has a low dispersion $\mathrm{D}(|\mathrm{D}|<5 \mathrm{ps} / \mathrm{nm} / \mathrm{km})$ in the wavelength range between 1.45 and $1.65 \mu \mathrm{m}$. According to our calculations, however, this particular structure is predicted to support two modes, as indicated from Fig.18(b).

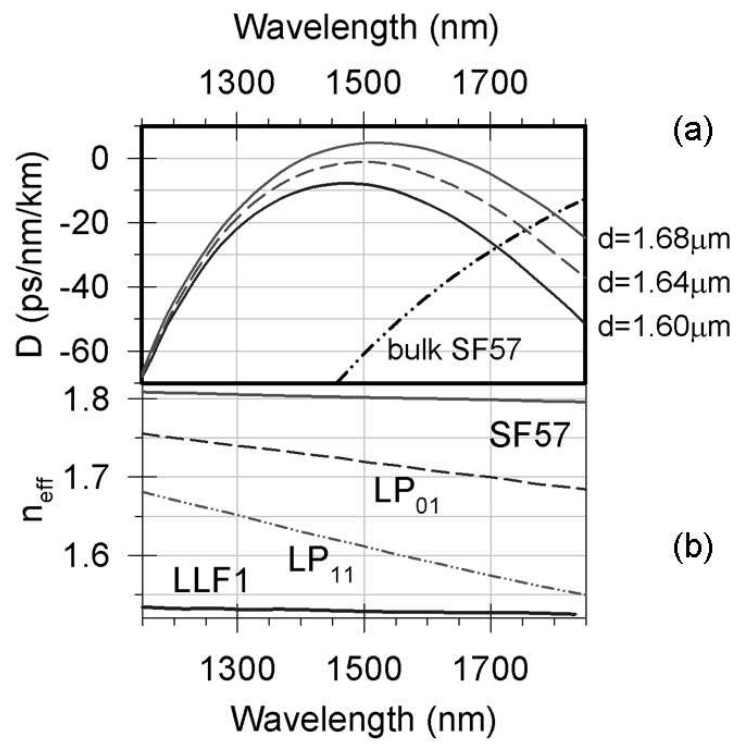

Fig. 18. Numerical simulation of (a) dispersion profile and (b) effective index $n_{\text {eff }}$ of the $L P_{01}$ and $L P_{11}$ modes of a SF57-LLF1 step-index fiber.

In order to obtain single-mode operation in such a step-index fiber, in analogy to the concept used in conventional W-type silica fibers with low index-contrast [43], an outer cladding with a higher index than the effective index of the $\mathrm{LP}_{11}$ mode is introduced in the fiber structure. By choosing an appropriate diameter ratio for the first and second cladding, it is possible to impose a very high confinement loss on the $\mathrm{LP}_{11}$ mode, thus enabling effectively single mode operation for a broad wavelength range. For the second cladding we chose Schott SF6 glass $(n=1.76$ at $1.55 \mu \mathrm{m})$, and found that the resulting SF57-LLF1-SF6 W-type fiber with $\mathrm{d}_{0}=1.68 \mu \mathrm{m}$ and $\mathrm{d}_{1}=7.4 \mu \mathrm{m}$ (see Fig.19), not only is single-moded but also has a dispersion profile very similar to the SF57-LLF1 step-index fiber with the same core diameter (shown in Fig.18(a)).

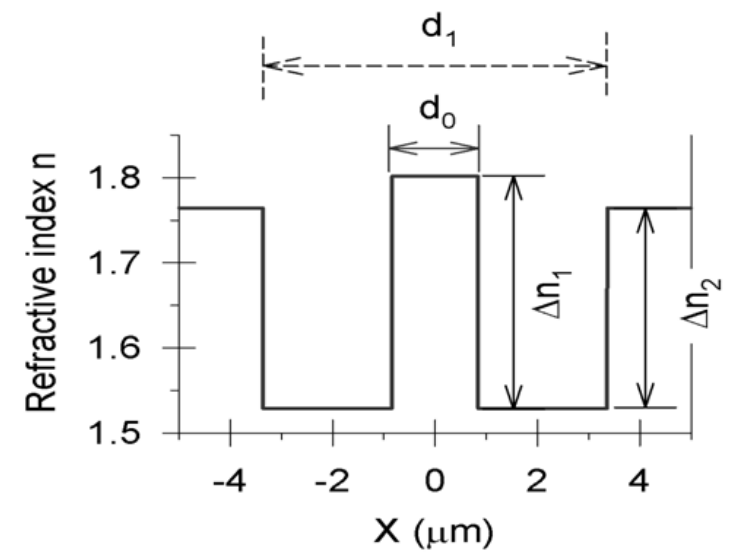

Fig. 19. Refractive index profile of the SF57-LLF1-SF6 W-type fiber.

In order to realize such a fiber design in practice, the crucial issue is whether the three proposed glasses exhibit sufficient thermal and chemical compatibility to allow for the fiber fabrication process. As already discussed previously and shown in Fig.5, the three glasses 
(Schott SF57, LLF1 and SF6) have a relatively large viscosity mismatch in the temperature range for fiber drawing (viscosities of $10^{6.5}-10^{4}$ poise), indicating a challenge for the fabrication. Based on previous experience and on preliminary tests, we proved however that with careful definition of the drawing conditions, fibers with good optical and mechanical properties could nonetheless be fabricated.

The W-type fiber was fabricated using the rod-in-tube method. The inner region was made from a SF57 rod with OD 2.9mm inserted into an LLF1 tube with an ID of 3mm and an OD of $13 \mathrm{~mm}$. The rod surface and both inner and outer surfaces of the tube were polished to optical quality. A SF57-LLF1 cane with $0.8 \mathrm{~mm}$ OD was then drawn from this assembly and inserted into a SF6 jacketing tube, from which the final fiber was drawn. Fig.20 shows SEM images of the cross-section of the fabricated fiber. The fiber had a core diameter of $1.63 \mu \mathrm{m}$ and a ratio of core diameter to cladding diameter of 1:4.4, the same as the value of the initial preform and matching exactly the original target. This straightforward achievement of structural targets clearly presents an advantage of our all-solid fiber technology over the holey fiber counterpart.

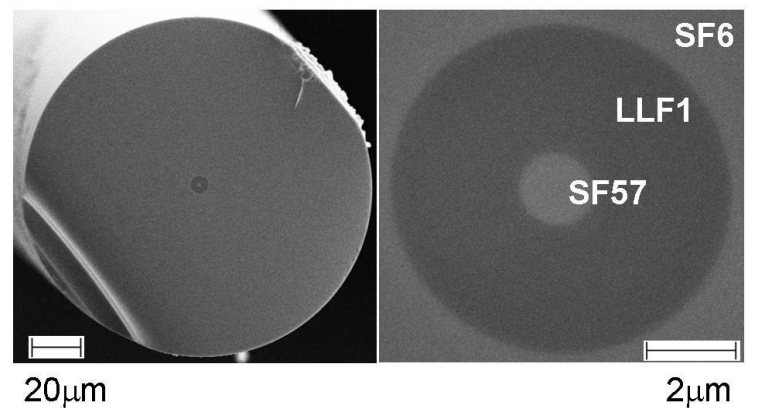

Fig. 20. SEM images of the $\mathrm{W}$-fiber with $\mathrm{d}_{0}$ of $1.63 \mu \mathrm{m}$.

The propagation loss of the fabricated fiber was measured to be $2.1 \pm 0.2 \mathrm{~dB} / \mathrm{m}$ at $1.55 \mu \mathrm{m}$ using the cutback method. We expect that this loss value can be reduced by further optimization of the fabrication process to a value similar to that of the 1D MOF described above. The predicted effective single mode guidance was confirmed by analyzing the near-field mode profiles of the fiber at $1.55 \mu \mathrm{m}$.

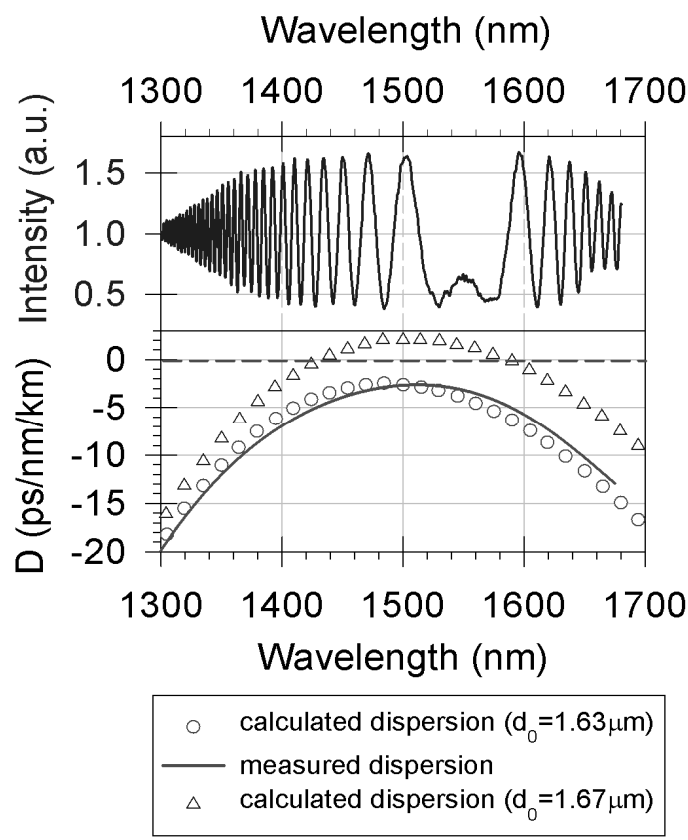

Fig. 21. (a) Observed interferometric pattern of single polarization broadband light propagating through the W-fiber; (b) measured and calculated dispersion of W-fibers with core diameters $\mathrm{d}_{0}$ of 1.63 and $1.67 \mu \mathrm{m}$. 
Despite the inherent difficulties in measuring the fiber's dispersion due to its low absolute value and short effective length, we obtained a very accurate and broadband measurement using a low-coherence Mach-Zehnder interferometric set-up and a supercontinuum source [44]. High quality interferograms were measured for the two fundamental polarization states, one of which is shown in Fig. 21(a), from 1.30 to $1.68 \mu \mathrm{m}$. The resulting dispersion curve, shown in Fig.21(b), is in excellent agreement with the numerical predictions, and shows a flattened profile at telecom wavelengths. In particular, at $\sim 1.52 \mu \mathrm{m}$ the dispersion slope is zero and $\mathrm{D}=-2.6 \mathrm{ps} / \mathrm{nm} / \mathrm{km}$. Fig.21(b) also shows that by drawing a $2 \%$ larger fiber, a region of small and flat anomalous dispersion a few hundreds of nm wide can be achieved.

The fiber effective nonlinearity $\gamma$ at $1.550 \mu \mathrm{m}$ was measured to be $820 \mathrm{~W}^{-1} \mathrm{~km}^{-1}$, in good agreement with a simulated value of $854 \mathrm{~W}^{-1} \mathrm{~km}^{-1}$ (effective area $\mathrm{A}_{\mathrm{eff}} \approx 2 \mu \mathrm{m}^{2}$ ). Note that this value is 1.6 times higher than the predicted nonlinearity of the SF57 dispersion flattened HF (zero dispersion and zero dispersion slope at $1.55 \mu \mathrm{m}$ ) discussed in Section 3.2, which shows a second advantage of the present design as compared to its air/glass counterpart.

We performed further FWM-based wavelength conversion experiments using this latest fiber, achieving markedly improved results in comparison to those obtained with the 1D MOF described in the previous Section. In these experiments a quasi-CW pump was placed in the middle of the C-band, $\left(\lambda_{\text {pump }}=1550 \mathrm{~nm}\right)$ while the $\mathrm{CW}$ signal was tuned across the entire range allowed by the sources we were using $(1530-1570 \mathrm{~nm})$. Fig.22 shows the results obtained for a peak pump power of $35 \mathrm{dBm}$ and a fiber length of $2.2 \mathrm{~m}$. A nearly flat conversion profile was obtained across the full $40 \mathrm{~nm}$ span that was available from our sources, and a conversion efficiency (defined as the ratio between the idler and signal power) of OdB was measured. Fig.22 also shows how the experimental results we obtained match the simulated gain profile, which suggests an overall $3 \mathrm{~dB}$ conversion bandwidth in excess of $60 \mathrm{~nm}$ can be achieved in this length of fiber.

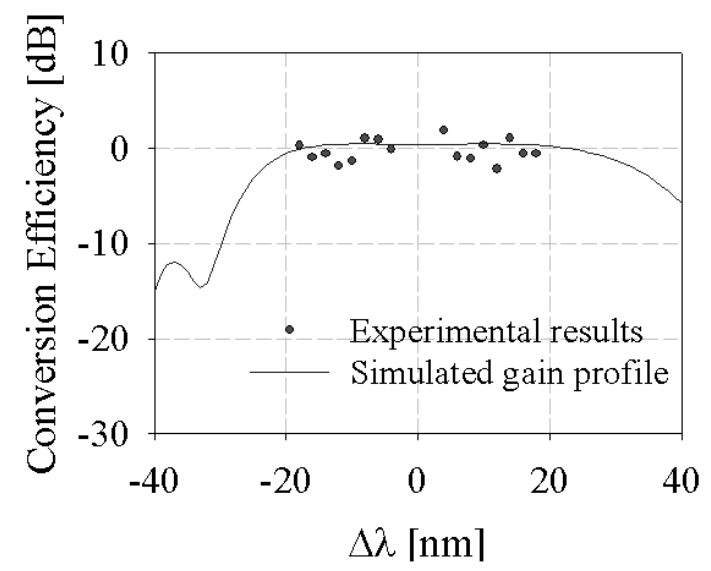

Figure 22: Measured (red symbols) and simulated (solid blue line) curves for the FWM conversion efficiency in the W-type fiber.

Using the same sample of the fabricated W-type fiber we have also experimentally demonstrated the simultaneous wavelength conversion of three $40 \mathrm{Gbit} / \mathrm{s}$ Differential Phase Shift Keyed (DPSK) signals [45]. The unique fiber properties allowed for a uniform conversion efficiency between the three wavelength converted signals of $\sim-12 \mathrm{~dB}$ and an excellent performance in terms of their noise properties as confirmed through eye diagram and bit error ratio measurements.

Finally, using a 3-m long sample of the same fiber we have demonstrated the generation of high quality, high repetition rate pulses $(>160 \mathrm{GHz})$ based on parametric mixing of two phaselocked narrow-linewidth tones and numerically investigated the potential of using such a fiber 
for the generation of even higher repetition rates $(>1 \mathrm{THz})[46]$.

\section{Conclusion}

We have reviewed our recent progress in the development of highly nonlinear lead silicate glass fibers with a tailored near-zero dispersion profile at telecommunication wavelengths. Investigated fiber types include: (i) air-suspended core holey fibers, (ii) holey fibers with complex holey cladding, (iii) all-solid 1D microstructured fibers, and (iv) W-type step-index fibers with high index-contrast. Various aspects relevant to the choice of fiber structure and of the glass materials were discussed in detail, with particular emphasis on the implications for the fiber fabrication. The conclusions are summarized in the following.

(i) In Air-Suspended Core HFs, the maximum theoretical nonlinearity $\gamma$ for a given glass can be achieved, as well as a ZDW at $1.550 \mu \mathrm{m}$, although in general with the penalty of a very high dispersion slope. The position of the ZDW is strongly dependent on the core diameter and also on the fine details of the core shape, indicating that, though not impossible, it is practically very challenging to engineer a precise dispersion profile around $1.55 \mu \mathrm{m}$ with such a fiber design.

(ii) In small core HFs based on a triangular lattice of holes, a flattened and near-zero dispersion profile can in principle be achieved in the $1.55 \mu \mathrm{m}$ region. However, several rings of holes, or complex double clad structures are required in order to obtain low confinement loss. Again this requirement makes it very challenging to precisely control the hole spacing $\Lambda$ and the hole-to-spacing ratio $\mathrm{d} / \Lambda$ to the degree of accuracy required in order to achieve the target dispersion profiles. We believe that this approach is more promising than the ASC HF, and is especially interesting for wavelength ranges where suitable pairs of compatible glasses cannot be found. Further improvements of the fabrication process are required in order to achieve improved control in fibers with a complex structure.

(iii) All-solid 1D MOFs with a coaxial-ring structure can achieve sub-dB/m attenuation levels at $1.55 \mu \mathrm{m}$, among the lowest ever reported for lead silicate glasses fibers, by utilizing finely polished glass discs and optimized fabrication conditions. In contrast to the small core HF, it is easy to accurately preserve the relative size of the high/low index features in the all-solid MOFs during the various stages of fabrication, i.e. going from preform, to cane, and finally to fiber. A difficulty is represented by the fact that the initial aspect ratio of the glass discs is not faithfully reproduced in the extruded preform, and suitable correction factors are required.

(iv) High index-contrast, W-type fibers provide the best compromise between fiber nonlinearity and accurate control of fiber structure amongst all the designs described here. We have developed a fabrication technique that allows for an extremely precise (submicron) control of the fiber structure. Consequently, control of chromatic dispersion to the $\mathrm{ps} / \mathrm{nm} / \mathrm{km}$ level can be achieved in this fiber, and flattened and near-zero dispersion profile around $1.55 \mu \mathrm{m}$ have been demonstrated. Reasonably low loss of $2.1 \mathrm{~dB} / \mathrm{m}$, high nonlinearity of $820 \mathrm{~W}^{-1} \mathrm{~km}^{-1}$, and singlemode operation have been achieved. Thus, these fibers offer an overall better performance as compared to all the other fiber types, including the coaxial all-solid 1D MOFs. Numerical simulations predict that fibers with a few percent larger core diameter than the fiber reported here would achieve a flattened and near-zero dispersion profile with around 200nm bandwidth of anomalous dispersion in the telecom window, opening up the possibility for a host of applications. The fabricated 1D MOF and the W-type fiber for FWM-based frequency were used for frequency conversion experiments at $1.55 \mu \mathrm{m}$, indicating the great potential of non-silica highly nonlinear fibers for applications in all optical processing at telecommunication wavelengths.

In addition to working toward improving the various steps involved in the fabrication of the fibers presented here, we believe future work should also be targeted at other crucial aspects, such as (i) reducing the fiber loss by lowering the impurities in the glass and bulk/interface defects in the fabrication, (ii) connecting the fabricated non-silica glass fiber with commercial 
silica fiber, e.g. by fusion splicing or mechanical splicing techniques, in order to improve the usability of such fibers, and (iii) explore different combinations of glasses offering higher nonlinearity.

\section{Acknowledgements}

The research leading to these results has received funding in part from the Engineering and Physics Science Research Council (UK) and the European Communities Seventh Framework FP/2007-2013 under grant agreement 224547. Dr. Francesco Poletti gratefully acknowledges support from the Royal Society through a University Research Fellowship.

\section{References}

[1] J.M. Dudley, and J.R. Taylor (Ed.), Supercontinuum Generation in Optical Fibers, Cambridge University Press, 2010.

[2] J. Hansryd, P.A. Andrekson, M. Westlund, J. Li, P.-O. Hedekvist, Fiber-based optical parametric amplifiers and their applications, IEEE J. Selected Topics in QE, 8 (2002). 506-520.

[3] J.C. Knight, T.A. Birks, P.St.J. Russell, and D.M. Atkin, All-silica single-mode fibre with photonic crystal cladding, Opt. Lett., 21 (1996) 1547-49.

[4] T.M. Monro, D.J. Richardson, N.G.R. Broderick, and P.J. Bennett, Holey optical fibres: an efficient modal model, J. Lightwave Technol., 17 (6) (1999) 1093-1102.

[5] T.M. Monro, Y.D. West, D.W. Hewak, N.G.R. Broderick, D.J.R. Richardson, Chalcogenide holey fibres, Elec. Lett., 36 (2000) 1998-2000.

[6] X. Feng, A.K. Mairaj, D. Hewak, T.M. Monro, Nonsilica glasses for holey fibers, J. Lightwave Technol., 23 (6) (2005) 2046-54.

[7] H. Nasu, O. Sugimoto, J. Matsuoka, and K. Kamiya, Non-resonant-type third-order optical nonlinearity of alkali silicate and alkali aluminosilicate glasses-contribution of individual chemical species in the glasses to $\chi^{(3)}$, J. Noncryst. Solids, 182 (1995) 321-27.

[8] I. Thomazeau, J. Etchepare, G. Grillon, and A. Migus, Electronic nonlinear optical susceptibilities of silicate glasses, Opt. Lett., 10 (1985) 223-225.

[9] R. Adair, L.L. Chase, and S. A. Payne, Nonlinear refractive index measurements of glasses of optical crystals, Phys. Rev. B, Condens. Matter, 39 (1989) 3337-50.

[10] D. Marcuse, Light transmission optics, (Van Nostrand Reinhold, New York, 1982), Chap. 8 and 12.

[11] D. W. Pohl and P. F. Meier, Multiphonon Absorption in NaF, Phys. Rev. Lett. 32 (1974) 58-61.

[12] I. H. Malitson, Interspecimen Comparison of the Refractive Index of Fused Silica, J. Opt. Soc. Am. 55 (1965) 1205-1209.

[13] T. Miyashita, T. Manabe, 'Infrared Optical fibers,' IEEE Trans. Microwave Theory and Techniques, MTT-30 (10) (1982) 1420-1438.

[14] Schott E-catalog Optical Glass 2000.

[15] G. Ghosh, 'Sellmeier coefficients and chromatic dispersions for some tellurite glasses,' J. Am. Ceram. Soc. 78(10) (1995) 2828-2830.

[16] W. Dumbaugh, J. Lapp, 'Heavy-metal oxide glasses,' J. Am. Ceram. Soc., 75(9) (1992) 2315-2326.

[17] S. Fujino, H. Ijiri, F. Shimizu, and K. Morinaga, "Measurement of viscosity of multi-component glasses in the wide range for fibre drawing”, J. Jpn. Instrum.Met., 62 (1998) 106-110. 
[18] Friberg, S.R., and Smith P.W., Nonlinear optical glasses for ultrafast optical switches, IEEE J Quantum Electron.,. QE-23 (1987) 2089-2234.

[19] D. N. Nikogosyan, Properties of Optical and Laser-related Materials: A Handbook, (John Wiley \& Sons, 1997).

[20] T. M. Monro, K. M. Kiang, J. H. Lee, K. Frampton, Z. Yusoff, R. Moore, J. Tucknott, D. W. Hewak, H. N. Rutt and D. J. Richardson, "High nonlinearity extruded single-mode holey optical fibers," OFC2002 (OSA, Washington, DC, 2002), Postdeadline FA1, 1-3, (2002).

[21] V. V. R. K. Kumar, A. K. George, W. H. Reeves, J. C. Knight, P. St. J. Russell, F. G. Omenetto, and A. J. Taylor, "Extruded soft glass photonic crystal fiber for ultrabroad supercontinuum generation," Opt. Express, 10 (2002) 1520-1525.

[22] P. Petropoulos, H. Ebendorff-Heidepriem, V. Finazzi, R.C. Moore, K. Frampton, D.J. Richardson, T.M. Monro, "Highly nonlinear and anomalously dispersive lead silicate glass holey fibers," Opt. Express, 11 (2003) 3568-3573.

[23] H. Ebendorff-Heidepriem, P. Petropoulos, S. Asimakis, V. Finazzi, R.C. Moore, K. Frampton, F. Koizumi, D.J. Richardson, T.M. Monro, “Bismuth glass holey fibers with high nonlinearity,” Opt. Express 12 (2004) 5082-5087.

[24] Shahraam Afshar V., Wen Qi Zhang, Heike Ebendorff-Heidepriem, and Tanya M. Monro, "Small core optical waveguides are more nonlinear than expected: experimental confirmation," Opt. Lett. 34, (2009) 3577-3579.

[25] J. Y. Y. Leong, P. Petropoulos, J. H. V. Price, H. Ebendorff-Heidepriem, S. Asimakis, R. C. Moore, K. E. Frampton, V. Finazzi, X. Feng, T. M. Monro, and D. J. Richardson, "High-nonlinearity dispersion shifted leadsilicate holey fibers for efficient 1- $\mu$ m pumped supercontinuum generation,” J. Lightwave Technol. 24 (2006) 183190.

[26] H. Ebendorff-Heidepriem, S. C. Warren-Smith, and T.M. Monro, "Suspended nanowires: Fabrication, design and characterization of fibers with nanoscale cores," Opt. Express, 17 (2009) 2646-2657.

[27] L. Dong, B. K. Thomas, and L. Fu, "Highly nonlinear silica suspended core fibers," Opt. Express, 16 (2008) 16423-16430.

[28] B. Elliott, M. Gilmore, Fiber Optic Cabling, Second Edition, (Newnes 2002) 55-56.

[29] S. Asimakis, P. Petropoulos, F. Poletti, J. Y. Y. Leong, R. C. Moore, K. E. Frampton, X. Feng, W. H. Loh, and D. J. Richardson, "Towards efficient and broadband four-wave-mixing using short-length dispersion tailored lead silicate holey fibers," Opt. Express 15 (2007) 596-601.

[30] K. Inoue, Experimental study on channel crosstalk due to fiber four-wave mixing around the zero-dispersion wavelength, J. Lightwave Technology, 12(6) (1994) 1023-1028.

[31] J.Y.Y. Leong, S. Asimakis, F.Poletti, P.Petropoulos, X. Feng, R.C.Moore, K.E.Frampton, T.M.Monro, H.Ebendorff-Heidepriem, W.H.Loh, D.J.Richardson, Towards zero dispersion highly nonlinear lead silicate glass holey fibres at 1550nm by structured-element-stacking, ECOC 2005 Glasgow 25-29 Sep 2005 Th4.4.5 (Postdeadline) [32] Francesco Poletti, Kentaro Furusawa, Zulfadzli Yusoff, Neil G. R. Broderick, and David J. Richardson, Nonlinear tapered holey fibers with high stimulated Brillouin scattering threshold and controlled dispersion, J. Opt. Soc. Am. B, 24(9) (2007) 2185-2194.

[33] F. Poletti, V. Finazzi, T. M. Monro, N. G. R. Broderick, V. Tse, and D. J. Richardson, "Inverse design and fabrication tolerances of ultra-flattened dispersion holey fibers," Opt. Express 13 (2005) 3728-3736.

[34] H. Ebendorff-Heidepriem and T.M. Monro, Extrusion of complex preforms for microstructured optical fibers, Opt. Express, 15(23) (2007) 15086-15092. 
[35] X.Feng, A.Camerlingo, F.Poletti, P.Petropoulos, K.E.Frampton, N.M.White, C.J.Oton, W.H.Loh, D.J.Richardson, Highly nonlinear non-silica glass microstructured optical fibers with near-zero dispersion slope for 1.55 micron applications, CLEO/Europe-EQEC 2009 Munich 14-19 Jun 2009 CE3.4

[36] A. Boskovic, S. V. Chernikov, J. R. Taylor, L. Gruner-Nielsen, and O. A. Levring, "Direct continuouswave

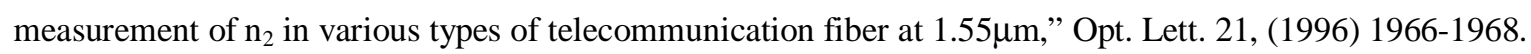

[37] X. Feng, T. M. Monro, P. Petropoulos, V. Finazzi, D. Hewak, "Solid microstructured optical fiber," Opt. Express, 11(18) (2003) 2225-30.

[38] X. Feng, T. M. Monro, P. Petropoulos, V. Finazzi, D. J. Richardson, "Extruded single-mode high-index-core one-dimensional microstructured optical fiber with high index-contrast for highly nonlinear optical devices," Appl. Phys. Lett. 87, (2005) 81110.

[39] X. Feng, F. Poletti, A. Camerlingo, F. Parmigiani, P. Horak, P. Petropoulos, W.H. Loh, and D. J. Richardson, Dispersion-shifted all-solid high index-contrast microstructured optical fiber for nonlinear applications at $1.55 \mu \mathrm{m}$, Opt. Express 17(22) (2009) 20249-20255.

[40] T. Hasegawa, T. Nagashima, N. Sugimoto, "Determination of nonlinear coefficient and group-velocity dispersion of bismuth-based high nonlinear optical fiber by four-wave-mixing," Opt. Commun., 281, (2008) 782787.

[41] A. W. Snyder, and J. D. Love, Optical waveguide theory (Chapman and Hall, London ; New York, 1983).

[42] X. Feng, G. M. Ponzo, F. Poletti, A. Camerlingo, F. Parmigiani, M. N. Petrovich, P. Petropoulos, N. M.White, W. H. Loh, D. J. Richardson, A Single-mode, High Index-contrast, Lead Silicate Glass Fibre with High Nonlinearity, Broadband Near-zero Dispersion at Telecommunication Wavelengths, submitted to ECOC 2010.

[43] T. Miya, K. Okamoto, Y. Ohmori, and Y. Sasaki, Fabrication of Low Dispersion Single-Mode Fibers Over a Wide Spectral Range, IEEE J. Quan. Electron., QE-17(6) (1981) 858-861.

[44] H. Shang, Chromatic dispersion measurement by white-light interferometry on metre-length single-mode optical fibres, Electron. Lett. 17(17) (1981) 603-605.

[45] A.Camerlingo, F.Parmigiani, X.Feng, F.Poletti, P.Horak, W.H.Loh, P.Petropoulos, D.J.Richardson, Multichannel wavelength conversion of $40 \mathrm{Gbit} / \mathrm{s}$ NRZ DPSK signals in a highly nonlinear dispersion flattened lead silicate fibre, Nonlinear Photonics 2010 Karlsruhe Germany 21-24 Jun 2010, NThA3.

[46] A. Camerlingo, F. Parmigiani, R. Slavik, X. Feng, F. Poletti, P. Horak, W. H. Loh, P. Petropoulos, D. J. Richardson, Generation of ultra-high repetition rate pulses in a highly nonlinear dispersion-tailored compound glass fibre, IEEE Summer Topical 2010, Cancun, Mexico, 19-21 Jul 2010, WC4. 


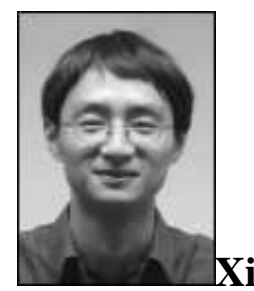

Xian Feng was born in Hangzhou, China. He received his Doctor of Engineering, majoring in Material Science, from Shanghai Institute of Optics \& Fine Mechanics, Chinese Academy of Sciences in 1999. He joined the soft-glass group of Optoelectronics Research Centre, University of Southampton in 2001, after the experience as the post-doctoral researcher in Kyoto University, Japan and Rutgers, The State University of New Jersey. His current research interests include the fabrication and the applications of microstructured specialty optical fibers based on non-silica optical glasses.

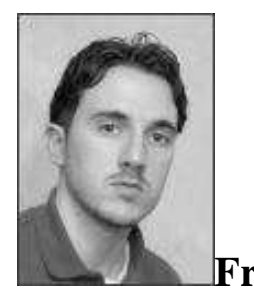

Francesco Poletti graduated with honours in Electronics Engineering at the University of Parma (Italy) in 2000. From 2000 to 2003 he worked on the design of optical telecoms networks at Marconi Communications (Italy and UK), and in 2007 he obtained a PhD in optoelectronics from the Optoelectronics Research Centre, University of Southampton (UK) for research on the direct and inverse design of microstructured optical fibers. His research, supported by a Royal Society University Research Fellowship, currently focuses on the study of micro and nano-structured optical waveguides, on the modeling of photonic bandgap structures, nonlinear optical processes in gas, liquid and semiconductor filled holey fibers, and on inverse electromagnetic problems.

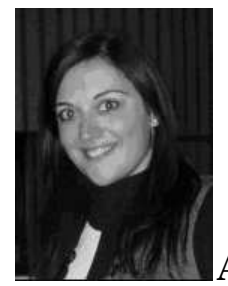

Angela Camerlingo was born in Naples, Italy. She graduated with honours in Electronics Engineering at the Second University of Naples in 2005. From January 2006 to July 2006 she worked at the Department of Electrical Engineering and Information Technology, Second University of Naples. Her research included the development of optical-fiber based pressure sensors. In October 2006 she joined the Optoelectronics Research Centre, University of Southampton, (UK), where she is currently working towards her Ph.D. degree in optical communication systems. Her current research interests include the development of optical fiber technologies and their applications for highly nonlinear systems. 
Francesca Parmigiani was born in Milan, Italy. She graduated with honours in Electronic Engineering at Politecnico di Milano, Milano, Italy, in 2002, and received the Ph.D. degree in optical communication systems at the Optoelectronics Research Centre (ORC), University of Southampton, Southampton, U.K in 2006. She is currently a Research Fellow at the ORC. In April 2010 she was awarded a prestigious Postdoctoral Research Fellowships from the Royal Academy of Engineering, in support of her research on the combination of all-optical signal processing and advanced modulation formats. Her research interests include ultra-fast alloptical sampling techniques, pulse shaping using specialized fiber Bragg gratings, all-optical nonlinear processing and switches mainly in optical fibers, as well as advanced modulation formats. Dr Parmigiani is a member of the Optical Society of America (OSA).

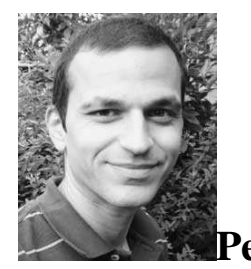

Periklis Petropoulos was born in Patras, Greece. He graduated from the Department of Electrical Engineering and Information Technology, University of Patras, in 1995, received the M.Sc. degree in communications engineering from the University of Manchester Institute of Science and Technology, Manchester, U.K., in 1996, and the Ph.D. degree in optical telecommunications from the Optoelectronics Research Centre (ORC), University of Southampton, Southampton, U.K. in 2000. His research interests include all-optical processing and switching in optical fibers; pulse manipulation for optical communications using fiber Bragg gratings, including applications in optical correlation systems for the implementation of optical code division multiple access and optical packet switched systems; silica and compound glass holey fibers and their nonlinear applications; and fiber lasers. His research has produced more than 240 papers in journals and conferences in the field of optical physics and optical communications. He is currently a Reader at the ORC.

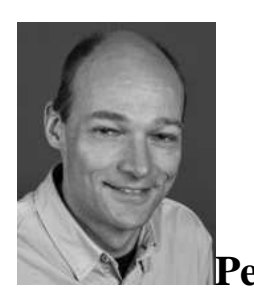

Peter Horak obtained a MSc degree in theoretical physics in 1993 and a $\mathrm{PhD}$ degree in theoretical quantum optics in 1997 from the University of Innsbruck, Austria. He held research positions at the University of Innsbruck, at the Ecole Normale Superieure in Paris, France, and at the University of Strathclyde in Glasgow, UK, before joining the ORC in 2001 where he is now a Senior Research Fellow. He heads the Computational Nonlinear Optics group at the ORC, focusing on theory and modeling of nonlinear and quantum optical systems. He currently holds a Research Council UK Academic Fellowship. 
Giorgio M. Ponzo was born in Tourin, Italy. He received a BSc degree (2006) and a MSc degree (2008) with honors in electronic engineering from the University of Palermo, Italy. $\mathrm{He}$ worked on the development of a DC/DC converter for VRM applications at ST Microelectronics (STM), Catania, Italy, for four months during his BSc degree thesis, and on the development of an innovative DC/DC converter for telecom applications, at Texas Instrument (TI), Freising, Germany, for six months during his MSc degree thesis. In 2008 he held a research position at University of Palermo working on high efficient DC/DC conversion. From the end of 2009 he is a Ph.D student at the Optoelectronics Research Centre (ORC), Southampton, UK. His research currently focuses on the development and characterization of microstructured optical fibers for several applications.

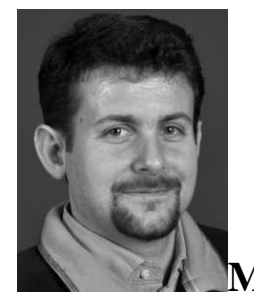

Marco Petrovich received a 'Laurea' degree in experimental Solid State Physics from the University of Padua, Italy in 1998 and a $\mathrm{PhD}$ in Optoelectronics from the University of Southampton in 2003, for work on the fabrication of chalcogenide fibers based on gallium lanthanum sulphide glass. In 1999 was a research assistant at the INFM MDM Labs working on characterisation thin insulator and conductive layers for MOS technology. He currently holds a position as Senior Research Fellow at the ORC, which he joined as a research staff in 2003. His research interests encompass fiber fabrication technologies, the development of novel silica, non silica and composite microstructured fibers, and the application of such fibers for nonlinear optics, novel laser sources, gas sensing and spectroscopy. He has authored or co-authored over 80 journal and conference papers. Dr Petrovich is a member of the Optical Society of America.

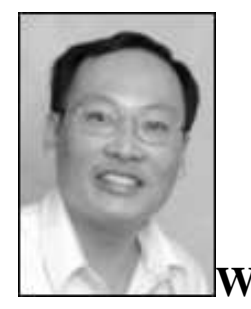

Wei H. Loh obtained his PhD in Electrical Engineering from Cornell University, NY. From 1991 to 1997, he was with the Optoelectronics Research Centre at University of Southampton. As a Senior Research Fellow working on application-specific fiber-optic devices, he made pioneering contributions to the design and fabrication of fiber Bragg gratings , dispersion compensation, semiconductor saturable absorbers for short pulse fiber lasers and soliton transmission, rare earth-doped fiber laser dynamics, distributed feedback fiber lasers and fiber laser phase noise . From 1997-2003, he worked in industry with various companies. From 1997-1998, he was with QPS Technology/Bragg Photonics (Montreal), where he oversaw the R\&D program for the commercialisation of fiber Bragg gratings and grating array sensors for both the telecommunications and oil sensing industries. At the end of 1998, he joined E-Tek Dynamics (San Jose), which then merged with JDS Uniphase to become the largest optical component vendor in the global marketplace. As Director for New Product Development at JDSU, he oversaw the new product development programme at its Silicon Valley site in San Jose, 
including the formulation of technology roadmaps and the controlled introduction of new products to the market. The range of products developed included passive optical components (free space, fiber as well as planar waveguides), switches/attenuators, network monitors, and amplifiers. He has been a Principal Research Fellow with the ORC since 2004. He has over 100 refereed journal and conference publications, and 8 patents.

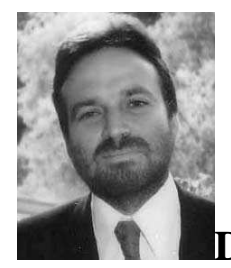

David J. Richardson was born in Southampton, U.K., in 1964. He received the B.Sc. degree and the Ph.D. degree in fundamental physics from Sussex University, U.K., in 1985 and 1989, respectively. In May 1989, he was a Research Fellow at the then recently formed Optoelectronics Research Centre (ORC), Southampton University, U.K. He is currently a Deputy Director at the ORC, responsible for much of the ORC's fiber-related activities. He is one of the founders of Southampton Photonics Incorporated, a university spin-off venture that has successfully commercialized elements of high-power laser technology developed within the ORC. His current research interests include amongst others: microstructured fibers, high-power fiber lasers, short pulse lasers, optical fiber communications, and nonlinear fiber optics. Prof Richardson has published more than 500 conference and journal papers in his time at the ORC, and produced over 20 patents. He is a frequent invited speaker at the leading international optics conferences in the optical communications, laser and nonlinear optics fields and is an active member of both the national and international optics communities. He was awarded a Royal Society University Fellowship in 1991 in recognition of his pioneering work on short-pulsed fiber lasers, and was made a Fellow of the Optical Society of America in 2005. He was elected as a Fellow of the Royal Academy of Engineering in recognition for his contributions to engineering in 2009. 